\title{
Periodic intensity distribution (PID) of mica polytypes: symbolism, structural model orientation and axial settings
}

\author{
Massimo Nespolo, ${ }^{a} \dagger \dagger$ Hiroshi Takeda, ${ }^{b}$ Toshiniro Kogure ${ }^{a}$ and Giovanni Ferraris ${ }^{c}$ \\ ${ }^{a}$ Mineralogical Institute, Graduate School of Science, University of Tokyo, 7-3-1 Hongo, Bunkyo-ku, Tokyo \\ 113-0033, Japan, ${ }^{b}$ Research Institute, Chiba Institute of Technology, 2-17-1 Tsudanuma, Narashino, Chiba 275-0016, \\ Japan, and ${ }^{c}$ Department of Mineralogical and Petrologic Sciences, University of Torino, via Valperga Caluso 35, \\ 10125 Torino, Italy.E-mail: nespolo@nirim.go.jp
}

(Received 1 September 1998; accepted 16 December 1998)

\begin{abstract}
Following a preliminary revisitation of the nomenclatures in use for mica polytypes, the properties of the periodic intensity distribution (PID) function, which represents the Fourier transform of the stacking sequence, are analysed. On the basis of the relative rotations of neighbouring layers, mica polytypes are classified into three types; for each type, the PID exists in different subspaces of the reciprocal space. A revised procedure to compute the PID, in which further restrictions on the structural model orientation are introduced, is presented. A unifying terminology based upon the most common symbols used to describe mica polytypes (RTW, Z and TS) is derived; these symbols represent the geometrical basis for the computation of the PID. Results are presented for up to four layer polytypes and are compared with the reflection conditions derived by means of Zvyagin's functions. Both the PID values and the reflection conditions are expressed in suitable axial settings and compared with previous partial reports, revealing some errors in previous analyses. A computer program to compute PID from the stacking symbols is available.
\end{abstract}

\section{Introduction}

Micas are phyllosilicates with general formula $I M_{2-3} T_{4} \mathrm{O}_{10} A_{2}$ (Rieder et al., 1998) (I: interlayer cations; $M$ : octahedral cations; $T$ : tetrahedral cations; $A=\mathrm{O}, \mathrm{OH}$, $\mathrm{F}, \mathrm{Cl})$. They are built by stacking a $2: 1$ or $T-O-T$ (tetrahedral-octahedral-tetrahedral) layer (Figs. 1 and 2 ) with interlayer cations between adjacent layers. The $T-O-T$ layer is commonly considered as a cleavage unit (Kogure, 1997) and polytypism arises from the stacking of this layer with $n \times 60^{\circ}$ rotations $(0 \leq n \leq 5)$ (Smith \& Yoder, 1956). Polytypes in which the position of any layer relative to the others and the transition from it to the adjacent layers are the same or equivalent for all

\footnotetext{
$\dagger$ Present address: National Institute for Research in Inorganic Materials, Research Center for Creating New Materials, 1-1 Namiki, Tsukuba-shi, Ibaraki 305-0044, Japan.
}

layers are called homogeneous polytypes (Zvyagin, 1988). The homogeneous polytypes were first derived by Smith \& Yoder (1956), who called them 'simple polymorphs'. By using Ramsdell (1947) notation, these polytypes are identified as $1 \mathrm{M}, 2 \mathrm{M}_{1}, 3 \mathrm{~T}, 2 \mathrm{M}_{2}, 2 \mathrm{O}$ and $6 \mathrm{H}$ [symbols according to Guinier et al. (1984)]. On the basis of the symmetry of the $O$ sheet, homogeneous polytypes have been divided into three families of MDO (maximum degree of order) polytypes (Backhaus \& Durovič, 1984; see below).

Several authors have used different symbols and approaches to deal with the problem of identifying the stacking sequence of mica polytypes. The most relevant results have been published in different journals and languages during the past forty years; therefore, they are briefly summarized below. The most powerful tool for unravelling the stacking sequence appears to be the comparison between the observed and calculated values of the periodic intensity distribution (PID) function.

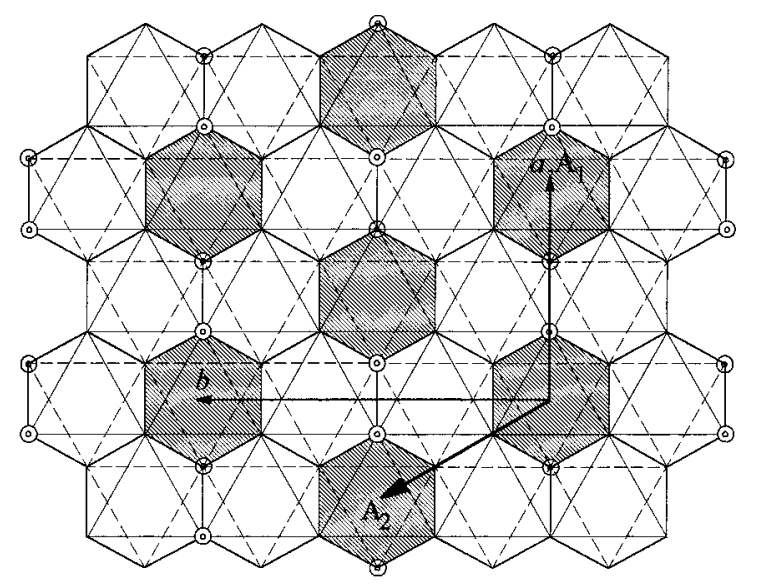

Fig. 1. The octahedral sheet of micas shown in (001) projection. The $M 1$ sites are shaded, $M 2$ sites are not shaded; concentric circles indicate $\mathrm{OH} / \mathrm{F}$ groups. $A_{1}$ and $A_{2}$ are hexagonal axes: they are rotated $30^{\circ}$ clockwise from the orientation given by Sadanaga \& Takeda (1969) and Takeda \& Sadanaga (1969). $a$ and $b$ are orthohexagonal axes, traced according to the standard orientation for Z symbols (Zhukhlistov et al., 1990); they correspond to the orthohexagonal cell $C_{1}$ as defined by Arnold (1996). 
This is the Fourier transform of the stacking sequence, obtained by removing the modulating effect of the layer transform from the structure factor (Takeda, 1967; Sadanaga \& Takeda, 1969; Takeda \& Sadanaga, 1969; Takeda \& Ross, 1995; see below). Hereinafter, the conditions under which PID can be obtained are revised, the computation procedure is redefined and a general correlation among the symbols involved is derived.

Hexagonal axes in the plane of the layer are henceforth indicated as $\left(A_{1}, A_{2}\right)$, whereas orthohexagonal axes are represented as $(a, b)$. Diffraction indices expressed in hexagonal axes are written as upper-case letters (HK.L), whereas those expressed in orthohexagonal or monoclinic axes are written as lower-case letters $(h k l)$. The orientation of the $(a, b)$ axes with respect to $\left(A_{1}, A_{2}\right)$ is that between the orthohexagonal $C_{1}$ cell and the hexagonal $P$ cell [see the work of Arnold (1996) and Nespolo, Takeda \& Ferraris (1997) and Nespolo et al. (1998)].†

\subsection{Choice of the unit layers}

Following Takéuchi (1971) and Takéuchi \& Haga (1971), the $T-O-T$ layer is henceforth called the M layer. Three translationally independent cationic sites exist in the $O$ sheet. One site is trans-coordinated by $\mathrm{OH} / \mathrm{F}$ and is labelled $M 1$; when the layer is centrosymmetric, the centre of symmetry is at this site. The remaining two sites are cis-coordinated by $\mathrm{OH} / \mathrm{F}$ and are labelled $M 2$; when the layer contains a symmetry plane, this plane relates the two $M 2$ sites (Fig. 1). Depending on the occupation of the $M$ sites, micas are divided into tri-octahedral and di-octahedral types. Then, on the basis of the distribution of the cations in these sites, the symmetry of the octahedral sheet can be $H(\overline{3}) 12 / m$ (homo-octahedral: the three sites are occupied by the same cation), $P(\overline{3}) 12 / m$ (meso-octahedral: one site is occupied by a cation different from that in the other two sites) or $P(3) 12$ (hetero-octahedral: all the three sites are differently occupied) (Ďurovič, 1994a) [layer-group notation after Dornberger-Schiff (1959), details given by Merlino (1990)]. In the so-called Pauling model (Pauling, 1930), the $T$ sheets have layer symmetry $P(6) \mathrm{mm}$. In the real structure, because of the difference in the lateral dimensions of the $T$ and $O$ sheets, tetrahedra rotate around $\mathbf{c}^{*}$ (Takéuchi \& Sadanaga, 1959) and the degree of rotation depends mainly upon the chemical composition and the temperature (Takeda \& Mori, 1970; Takeda \& Morosin, 1975; Catti et al., 1989). The model that takes into account this rotation is hereinafter called the trigonal model. The trigonal model is also known as the Radoslovich model (Rado-

$\dagger$ The orthohexagonal cell used since long ago in describing micas corresponds to $C_{1}$ (e.g. Donnay et al., 1964). Other authors, such as the OD school, adopted the $C_{2}$ cell (e.g. Durovič et al., 1984). This different choice of the orthohexagonal cell has to be taken into account when comparing the descriptions by different authors. slovich, 1961). It was proposed for the first time by Belov (1949). The tetrahedral rotation reduces the symmetry of the $T$ sheets to $P(3) 1 \mathrm{~m}$. The two $T$ sheets within a layer are staggered by $|a| / 3$ in (001) projection; therefore, in both models the ideal layer symmetry of the $\mathrm{M}$ layer is $C 12 / m(1)$ (homo- and some meso-octahedral micas) or $C 12(1)$ (the remaining meso-octhaedral and all hetero-octahedral micas) (Dornberger-Schiff et al., 1982). However, the symmetry of a layer may depend on its stacking in a polytypic structure; the deviation from the ideal symmetry has been called 'desymmetrization' (Durovič, 1979). Desymmetrization is commonly observed in $2 M_{1}$ and $3 T$ polytypes, where the layer symmetry is usually $C \overline{1}$ and $C 12(1)$, respectively (Pavese et al., 1997, 1998). In margarite-2 $M_{1}$, further desymmetrization to $C 1$ has been reported (Guggenheim \& Bailey, 1975, 1978). The space-group type can allow but does not force the desymmetrization; as a matter of fact, the highest layer symmetry $C 12 / m(1)$ was observed, within the limit of the accuracy of the structure refinement, in coexisting $1 M$ and $2 M_{1}$ meso-octahedral oxybiotites having truly polytypic character (Ohta et al., 1982).

The origin of the $\mathrm{M}$ layer is usually taken in the interlayer region; the location of the origin of the $O$ sheet in itself depends on the kind and distribution of the cations. It is always taken in the $M 1$ site for homooctahedral micas; however, in meso-octahedral micas it is in the site with different occupation and in heterooctahedral micas corresponds to the site containing the lowest electronic density (Durovič et al., 1984). For these micas, the origin of the $O$ sheet can thus be either in the $M 1$ site or in one of the two $M 2$ sites. As a consequence,

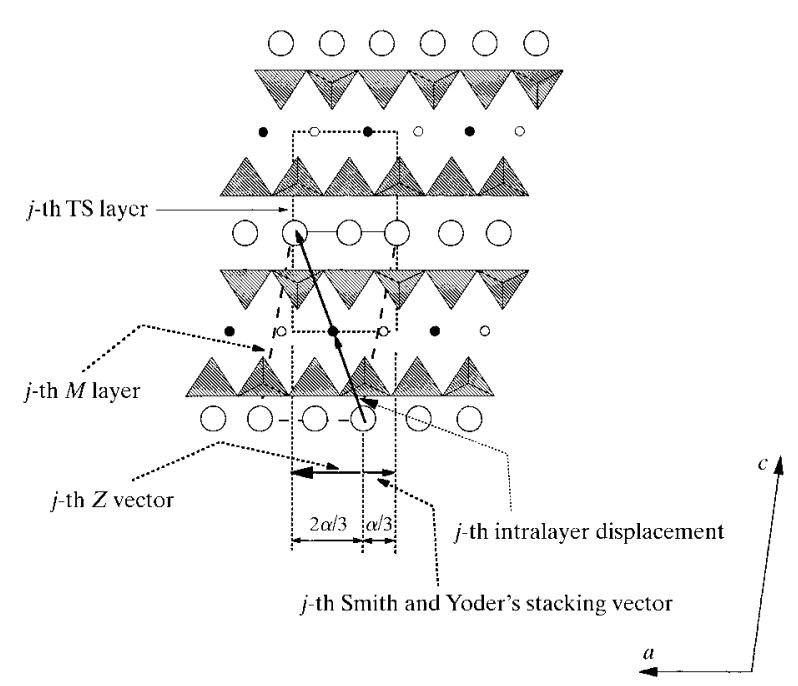

Fig. 2. Comparison between different unit layers for mica polytypes in projection along the $b$ axis. Different kinds of stacking vectors are shown. The scale along $\mathbf{c}$ is compressed. Black and open small circles represent $M 1$ and $M 2$ sites, respectively. Large open circles are interlayer cations. 
two kinds of $\mathrm{M}$ layer exist, which are hereinafter indicated as M1 and M2, respectively, when a distinction is necessary. Their highest layer symmetries are $C 12 / m(1)$ and $C 1(2) 1$, respectively (Zvyagin, 1974). These two layers can be distinguished only if their cation distribution is known. For example, hetero-trioctahedral $1 M$ micas have been refined by Backhaus (1983) (lepidolite) and Guggenheim \& Bailey (1977) (zinnwaldite) in the space group $C 2$. Both structures had the highest heterooctahedral layer symmetry $C 12(1)$, but the lepidolite was constructed by M1 layers whereas the zinnwaldite was built by M2 layers. Guggenheim (1981) refined a meso-octahedral lepidolite- $1 M$ in the space group $C 2$ with layer symmetry $C 12(1)$ : the cation distribution shows that the lower layer symmetry arises from the presence of the M2 layer and not from a desymmetrization of the M1 layer. The M1 layer is far more common. However, several examples of micas built by the M2 layer are known [Zvyagin et al., 1985; see references in: Durovič et al., 1984; Nespolo, 1998].

Although a single type of unit layer is sufficient to describe polytypism of micas, the choice of more than one type is suitable for some purposes. To show the OD ( $\mathrm{OD}=$ order-disorder $)$ character of mica polytypes, two kinds of OD layers and one kind of OD 'packet' (corresponding to half $\mathrm{M}$ layers) are used (DornbergerSchiff et al., 1982). To compute PID, Sadanaga \& Takeda (1969) and Takeda \& Sadanaga (1969) introduced four unit layers with idealized trigonal symmetry (TS unit layers), defined by two adjacent half M1 layers and the interlayer cations between them (Fig. 2). These layers are labelled $\mathrm{D}, \mathrm{D}^{*}[P(\overline{3}) 1 \mathrm{~m}$ symmetry for homo-octahedral micas], $\mathrm{T}$ and $\mathrm{T}^{*}[P(\overline{6}) 2 \mathrm{~m}$ symmetry for homooctahedral micas] and are staggered by means of $|a| / 3$ translations along one of the equivalent hexagonal axes. $\mathrm{D}$ is related to $\mathrm{D}^{*}$ and $\mathrm{T}$ to $\mathrm{T}^{*}$ by a $180^{\circ}$ rotation around c* (Fig. 3).

\subsection{Mica polytypes as $O D$ structures: family reflections}

Polytypes in which M layers are rotated by $2 n \times 60^{\circ}$ only are OD polytypes belonging to subfamily A (Backhaus \& Durovič, 1984) $\dagger$ and the coordination polyhedron for the interlayer cations is a trigonal antiprism. Subfamily A includes the three most common homogeneous (MDO) polytypes $\left(1 M, 2 M_{1}, 3 T\right)$ and most of the inhomogeneous polytypes reported to date. On the other hand, polytypes in which $M$ layers are rotated by $(2 n+1) \times 60^{\circ}$ only are OD polytypes belonging to subfamily B (Backhaus \& Durovič, 1984) and the coordination polyhedron for the interlayer cations is a trigonal prism. Only two homogeneous (MDO) polytypes belonging to subfamily $\mathrm{B}$ have been reported to date: $2 M_{2}$ (e.g. Takeda \& Burnham, 1969;

$\dagger$ Takeda \& Ross (1995) called ternary polytypes the OD subfamily A polytypes.
Černý et al., 1970; Takeda et al., 1971; Zhukhlistov et al., 1973) and $2 O$ (Giuseppetti \& Tadini, 1972; Filut et al., 1985). The remaining polytypes are called mixed-rotation polytypes (Nespolo, 1999); within the trigonal model, they contain both kinds of interlayer coordination polyhedra, violating the vicinity condition (Dornberger-Schiff, 1964), and thus are non-OD polytypes. They have been only seldom reported (Takeda, 1967; Bailey \& Christie, 1978; Rule et al., 1987; Kogure \& Nespolo, 1999a,b) and the relatively low quality of the structure refinement does not give information on the tetrahedral rotation angle. Backhaus \& Durovič (1984) suggested that this could be zero, thus corresponding to the Pauling model. In such a case, mixed-rotation polytypes would be OD polytypes too.

A Fourier series calculated with a three-dimensional subset of diffractions corresponding to a subgroup of translations in the reciprocal lattice gives a fictitious average structure, which is called a superposition structure. An $n$-fold superposition structure is obtained from a translation subgroup of order $n$ (DornbergerSchiff, 1964; Durovič, 1994b). Among the possible superposition structures, the one in which all the possible positions of all OD layers are simultaneously realized is of particular importance; it has also been called 'family structure' (Durovič, 1994b). The subset of the diffractions corresponding to the family structure are called family reflections; they are always sharp and are common to all the OD polytypes built on the same structural principle (Durovič \& Weiss, 1986). The remaining ones are called non-family reflections and are typical of each polytype; they are sharp or diffuse depending on whether the polytype is ordered or not (Durovič, 1992, 1997; Ďurovič \& Weiss, 1986; FichtnerSchmittler, 1979; Merlino, 1997). The family reflections indicate whether a polytype belongs to subfamily A or B or it is a mixed-rotation polytype. Then, non-family reflections belonging to the $(0 \mathrm{kl})$ plane (common to all polytypes with the same $b c$ projection: $C_{1}$ setting) are in principle enough to identify any polytype of mica, except homometric structures (Weiss \& Wiewióra, 1986; Durovič, 1992).

Within the Pauling model, the family reflections are those with $h=0(\bmod 3)$ and $k=0(\bmod 3)\left(C_{1}\right.$ setting $)$ and the family structure is ninefold (Weiss \& Wiewióra, 1986). These are the only family reflections for mixedrotation polytypes, but they are not useful for identification purposes because they are common to all polytypes of the same mineral group (Durovič, 1994b). On the other hand, within the trigonal model, the family reflections are those with $k=0(\bmod 3)$ and the family structure is threefold (Weiss \& Wiewióra, 1986). Family reflections corresponding to $h \neq 0(\bmod 3)$ show only one reflection out of $N(N$ is the number of layers of the polytype) in the case of subfamily A, but two equally spaced reflections in the case of subfamily B (Nespolo, 1999). 


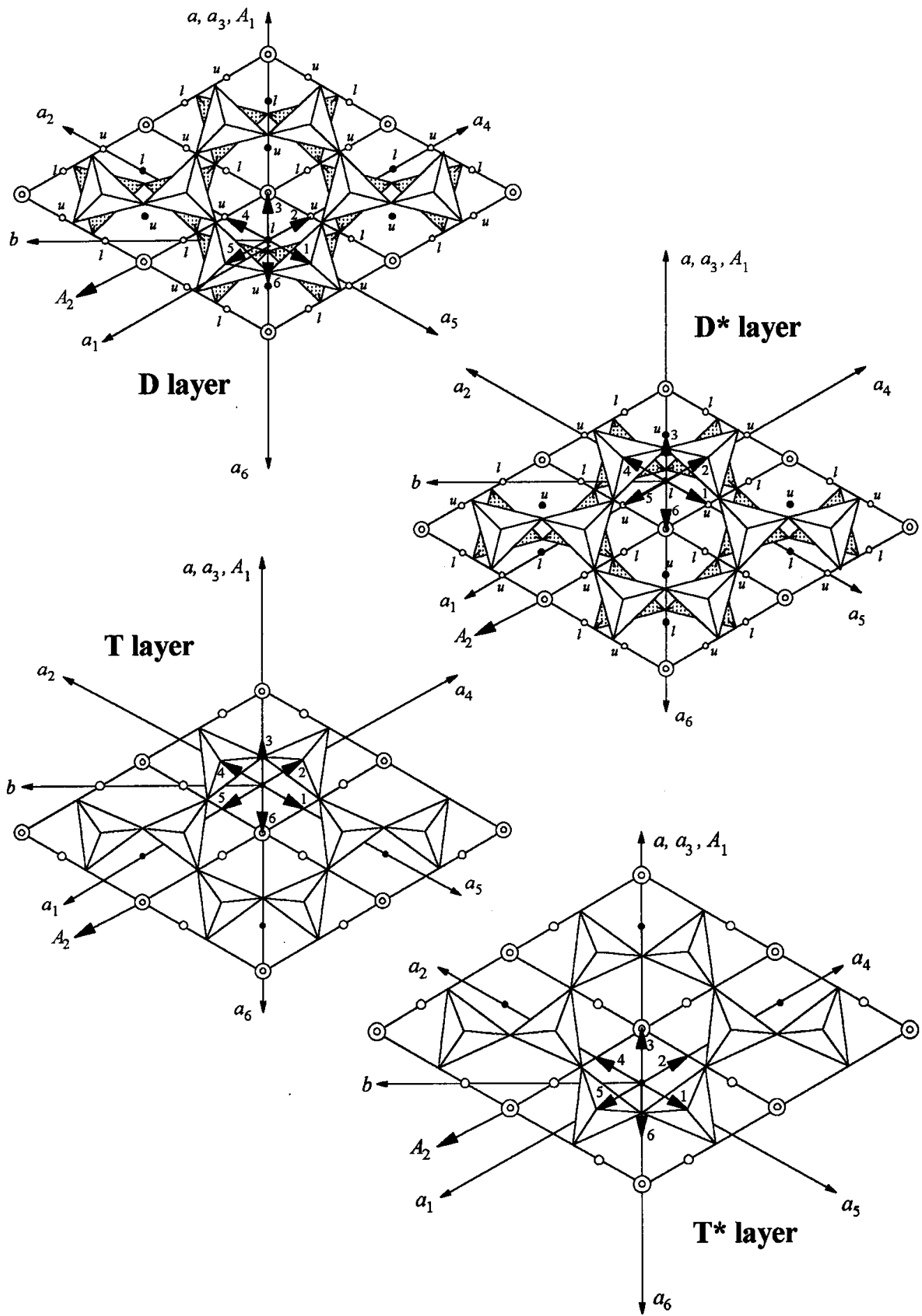

Fig. 3. The four TS unit layers. $a, b$ : orthohexagonal axes of the $C_{1}$ setting; $a_{1}-a_{6}$ : structure-related $a$ orthohexagonal axes in the six possible orientations (Zhukhlistov et al., 1990); $A_{1}, A_{2}$ : hexagonal axes. The six digits 1-6 indicate the intralayer displacement vectors: these are superimposed on the projection of each of the four TS unit layers. Since each TS layer is defined from the $O$ sheet of an M layer to the $O$ sheet of the successive M layer, two sets of $a_{1}-a_{6}$ axes should be considered, both with the origin in the $M 1$ site. These two sets define the orientation of the upper intralayer displacement vector of the lower M layer and of the lower intralayer displacement vector of the upper $\mathrm{M}$ layer. In order to improve the readability of the figure, only the first sets are shown. Black and open small circles represent $M 1$ and $M 2$ sites, respectively. Concentric circles represent overlapped interlayer cations and OH/F. $u$ and $l$ indicate octahedral cations with $z=+1 / 2$ and $z=-1 / 2$, respectively. For $\mathrm{T}$ and $\mathrm{T}^{*}$ layers these are overlapped in (001) projection. 


\subsection{Lattice features}

Orthogonal mica polytypes can be hexagonal (no examples known), trigonal and orthorhombic; the lattice of triclinic polytypes is metrically (pseudo-)monoclinic. Non-orthogonal polytypes are divided into Class $a$ and Class $b$ depending on whether the $c$ axis is inclined towards the shortest or towards the longest of the two axes in the plane of the layer (Nespolo et al., 1998). This classification corresponds to different values of the (001) projection of the $c$ axis, labelled $\mathbf{c}_{n}$ by Zvyagin (1997). In all mica polytypes, a pseudo-hexagonal lattice can be located, which is multiple for non-orthogonal polytypes; for Class $b$ polytypes this lattice is centred and a markedly pseudo-rhombohedral primitive cell exists (Nespolo, 1999). The number $N$ of layers building a polytype can be expressed as

$$
N=3^{n}(3 K+L)
$$

where $K$ and $n$ are non-negative integers and $L=1$ or 2 . $n$ defines the series and $L$ the subclass; $K$ is a constant entering in the transformation matrices relating axial settings (Nespolo et al., 1998).

\subsection{Identification of the stacking sequence}

The Fourier transform of a polytype is given by the Fourier transform of the stacking sequence, which is a fringe function (Lipson \& Taylor, 1958), modulated by the Fourier transform of the layer(s) (Takeda, 1967). The layer transform is a nondiscrete function in the direction without periodicity, i.e. that indicated in parentheses in the layer symbol. When the building layers are related by just an integral submultiple of the translation period, it is possible to extract their Fourier transform from the expression of the structure factor, which thus assumes the simple form of a product of the layer transform and of the stacking sequence transform (see below). This second term expresses the periodicity in reciprocal space appearing when a structure is built by translation of subunits. This was independently recognized by Takeda (1961) and, more indirectly, by Andreeva (1962). The periodicity in reciprocal space has been used to solve the structure of zinkenite $\left(\mathrm{PbS} \cdot \mathrm{Sb}_{2} \mathrm{~S}_{3}\right.$ ) (Takeda, 1961; Sadanaga \& Takeda, 1964; Takeda \& Horiuchi, 1971) and to identify minerals with a mixed-layer structure (D'yakonov, 1962, 1963). It has been successfully applied to identify the stacking sequences of polytypes of micas (Takeda \& Donnay, 1965; Ross et al., 1966; Takeda, 1967), of SiC (Tokonami \& Hosoya, 1965; Tokonami, 1966) and of $\mathrm{SiC}$ and $\mathrm{ZnS}$ (Farkas-Jahnke, 1966; Dornberger-Schiff \& FarkasJahnke, 1970; Farkas-Jahnke \& Dornberger-Schiff, 1970). The Fourier transform of $\mathrm{SiC}$ and of the $\mathrm{ZnS}$ unit is much different from the atomic scattering factors, and the periodicity is recognized without difficulty. In the case of micas, the unit layer is $10 \AA$ thick and the periodicity is not easily recognized. With the obvious exception of the $1 M$ polytype, subsequent layers are related by both translations and rotations. However, for a subspace of the reciprocal space, the Fourier transform of the M layer in the six possible orientations is almost unmodified (Takeda, 1967). Removal of the modulating effect of the layer gives the approximated Fourier transform of the stacking sequence. This is known as the periodic intensity distribution (PID) function (Takeda, 1967; Sadanaga \& Takeda, 1969; Takeda \& Sadanaga, 1969; Takeda \& Ross, 1995). Comparison of calculated and observed PID values along non-family reciprocallattice rows parallel to $\mathbf{c}^{*}$ is in principle sufficient to identify any mica polytype (Takeda \& Ross, 1995).

\section{Symbolic description and layer orientation}

General symbols to describe mica polytypes have been developed by the OD school (Dornberger-Schiff et al., 1982; Backhaus \& Ďurovič, 1984; Ďurovič et al., 1984; Weiss \& Wiewióra, 1986). However, for the purposes of the present research, three kinds of symbols are used here.

(i) Z symbols (Zvyagin et al., 1979; Zvyagin, 1985; Zhukhlistov et al., 1990) are oriented symbols giving the absolute orientation and relative displacement of half $\mathrm{M}$ layers. A space-fixed orthohexagonal reference is used, based on $(a, b)$ axes [ $C_{1}$ setting of Nespolo et al. (1998)]. The stacking sequence is indicated by the (001) projection of the intralayer displacement vectors, with length $|a| / 3$; these vectors connect the origin of the $O$ sheet with the nearest interlayer site and vice versa (the sequence of layers must be seen from the same direction). The six possible orientations of these vectors are indicated by six structure-related $a_{i}$ axes $(i=1-6)$. Their (001) projection is indicated by the $\mathrm{Z}$ symbol $i$ $(i=1,2, \ldots, 6)$ when the $a_{i}$ axis is parallel to the spacefixed axis $a$ (Figs. 2 and 3). The complete $\mathrm{Z}$ symbol is $i j 0 \mathrm{kl} 0 \mathrm{mn} \ldots$, where 0 indicates the only interlayer displacement vector in micas, whose (001) components are $(0,0)$ (because of the presence of interlayer cations, there is no relative displacement between two neighbouring M layers in micas). Symbols giving the orientation of the two halves of an M layer ( $i$ and $j, k$ and $l, m$ and $n$ etc.) must have the same parity in order to obtain an octahedral coordination for the cations. Besides, for micas built by M1 layers, $i=j, k=l, m=n$ etc.; the symbol 0 can be omitted and a shortened symbol $I K M \ldots$ is adopted (Zhukhlistov et al., 1990; see Fig. 1 therein). For micas built by M1 layers only, the complete displacement vectors ( $Z$ vectors, hereinafter) are thus simply obtained by taking twice the intralayer displacement vectors.

(ii) RTW symbols (Ross et al., 1966) are orientationfree rotational symbols giving the relative rotation between neighbouring $M$ layers; they can be thought of as differences between pairs of shortened $\mathrm{Z}$ symbols. RTW symbols are written as a sequence of $N$ digits 
Table 1. $Z$ symbols and $(a, b)$ components (expressed as multiples of 1/3) of the corresponding $Z$ vectors with respect to the space-fixed reference (Zvyagin, 1967)

$\begin{array}{llll}\text { Z symbol } & (a, b) \text { components } & \text { Z symbol } & (a, b) \text { components } \\ 3 & (\overline{1}, 0) & 6 & (1,0) \\ 4 & (1,1) & 1 & (\overline{1}, \overline{1}) \\ 5 & (\overline{1}, 1) & 2 & (1, \overline{1})\end{array}$

$A_{j}=0, \pm 1, \pm 2,3$, the $j$ th symbol giving the rotation angle between the $j$ th and $(j+1)$ th $\mathrm{M}$ layers as an integer multiple of $60^{\circ} . \dagger$

(iii) TS symbols (Sadanaga \& Takeda, 1969; Takeda \& Sadanaga, 1969) give the relative positions of TS unit layers by means of only displacements and no rotations. TS symbols are written as a sequence of $N$ symbols $L_{j}\left(\Delta X_{j}, \Delta Y_{j}\right), 1 \leq j \leq N$, where $L_{j}$ is the kind of layer, $\left(\mathrm{D}, \mathrm{D}^{*}, \mathrm{~T}, \mathrm{~T}^{*}\right)$ and $\left(\Delta X_{j}, \Delta Y_{j}\right)$ are the $\left(A_{1}, A_{2}\right)$ components of the shift vector between the $j$ th TS layer and the $N$ th TS layer of the previous repeat (Fig. 2).

Only Z symbols can distinguish between M1 and M2 layers. Since PID is computed by means of TS symbols (see below), the stacking sequence obtained from PID analysis is the one for homo-octahedral micas. Therefore, hereinafter the approximation $\mathrm{M} 1=\mathrm{M} 2=\mathrm{M}$ is called the homo-octahedral approximation.

The stacking sequence of mica polytypes is quite often described by means of the stacking vectors according to the original definition of Smith \& Yoder (1956), i.e. the (001) projection of vectors connecting two nearest interlayer cations on the two sides of a layer (Fig. 2). Unlike $\mathrm{Z}$ vectors, these vectors do not pass through any cation site in the $O$ sheet. Therefore, stacking vectors of Smith \& Yoder (1956) cannot distinguish between M1 and M2 layers and they too correspond to the homo-octahedral approximation. $\neq$

Both the M layer and the TS unit layers have the origin in the interlayer region. However, TS unit layers are shifted along [001] by half the thickness of the M layer (Fig. 2) (Sadanaga \& Takeda, 1969; Takeda \& Sadanaga, 1969); therefore, the origin of the $j$ th TS unit layer is in the interlayer region where the origin of the $(j+1)$ th $\mathrm{M}$ layer is located. Besides, the $j$ th TS unit layer is defined by the relation between the $j$ th and the $(j+1)$ th $\mathrm{M}$ layers: $\mathrm{D}$ and $\mathrm{D}^{*}$ layers appear in the middle of two M layers rotated by $2 n \times 60^{\circ}$ [i.e. the RTW symbol is $\left.A_{j}=0(\bmod 2)\right]$, while $\mathrm{T}$ and $\mathrm{T}^{*}$ layers appear

\footnotetext{
$\dagger$ It is now common to see RTW symbols written inside square brackets; their use was not specified by the authors in their original paper (Ross et al., 1966).

\$ Stacking vectors similar to those of Smith \& Yoder (1956) were introduced earlier by Dekeyser \& Amelinckx (1953) as the projection of the vector connecting two $\mathrm{OH} / \mathrm{F}$ sites within an $\mathrm{M}$ layer. A numerical symbolism similar to that of Zvyagin and co-workers was also introduced, which, however, considered whole M layers and not half M layers. The description by Dekeyser \& Amelinckx (1953) is correct in the homo-octahedral approximation.
}

in the middle of two $\mathrm{M}$ layers rotated $(2 n+1) \times 60^{\circ}[$ i.e. $\left.A_{j}=1(\bmod 2)\right]$.

The origin of Zvyagin's coordinate vectors is in the $O$ sheet, but $Z$ vectors are defined as the (001) projection of the vectors passing through the origin of the $O$-sheet site and connecting two interlayer sites below and above an M layer (Zvyagin et al., 1979). The $j$ th $\mathrm{Z}$ vector gives thus the $(a, b)$ components of the displacement between the $j$ th and the $(j+1)$ th $M$ layers (Fig. 2). As seen above, in the homo-octahedral approximation, $\mathrm{Z}$ vectors are just twice the intralayer displacement vectors; their $(a, b)$ components are given in Table 1.

The four TS unit layers, together with intralayer displacement vectors, are shown in Fig. 3. In these layers, the atoms have identical coordinates except those of $M_{O}$ and $O_{B}$, the hexagonal coordinates of which are presented in Table 2 (Sadanaga \& Takeda, 1969; Takeda $\&$ Sadanaga, 1969). The lower octahedral sheet of the $j$ th TS layer is the octahedral sheet of the $j$ th M layer. By labelling $o$ (odd) and $e$ (even) the orientation parity of $\mathrm{Z}$ symbols of half layers, the following correspondences with the complete $\mathrm{Z}$ symbols are obtained from Fig. 3:

$$
\mathrm{D}=o 0 o ; \quad \mathrm{D}^{*}=e 0 e ; \quad \mathrm{T}=e 0 o ; \quad \mathrm{T}^{*}=o 0 e .
$$

\section{PID in terms of TS unit layers}

Every mica polytype can be approximately described by means of one or more TS unit layers, with mutual staggers of $|a| / 3$ along one of the hexagonal axes (Sadanaga \& Takeda, 1969; Takeda \& Sadanaga, 1969), and two neighbouring TS layers must connect a half M layer with the same orientation parity. On the basis of equations (2), there are only eight possible pairs of TS unit layers (DD; $\mathrm{D}^{*} \mathrm{D}^{*} ; \mathrm{TT}^{*} ; \mathrm{T}^{*} \mathrm{~T} ; \mathrm{DT}^{*} ; \mathrm{D}^{*} \mathrm{~T} ; \mathrm{TD} ; \mathrm{T}^{*} \mathrm{D}^{*}$ ). Besides, in order to match the cation positions, the layer stacked over a $\mathrm{D}$ or $\mathrm{T}$ layer has to be shifted by $-a / 3$, whereas the layer stacked over a $\mathrm{D}^{*}$ or $\mathrm{T}^{*}$ layer has to be shifted by $+a / 3$ (Table 2). The Fourier transform of an $N$-layer mica polytype in terms of TS unit layers in hexagonal axes is expressed as [modified after Takeda \& Sadanaga (1969)]:

$$
\begin{aligned}
G^{N}(H K . L)= & \sum_{j=1}^{N} G_{j}\left(H K . L_{R}\right) \exp \left\{2 \pi i \left[H \Delta X_{j}+K \Delta Y_{j}\right.\right. \\
& +L(j-1) / N]\} .
\end{aligned}
$$

$G_{j}\left(H K . L_{R}\right)$ is the Fourier transform of the $j$ th TS unit layer, which is two-dimensionally periodic. In the direction without periodicity, the reciprocal-lattice coordinate is not restricted to integral values but is a real variable and is here labelled $L_{R}$. In equation (3), $G_{j}$ plays a role analogous to that of the atomic scattering factor in the expression of the structure factor.

As shown above, the atomic coordinates in the four TS unit layers differ only for $O_{B}$ and $M_{O}$, but within the 
Table 2. Hexagonal coordinates of the octahedral cations and basal oxygen atoms in the upper and lower halves of the four TS unit layers

The coordinates of symmetry-independent atoms only are given. The hexagonal axes within the layer are assumed to have $A_{1}$ coincident with $a$ of the space-fixed reference (see Fig. 3). $f(\alpha)$ and $f(\psi)$ are functions of the ditrigonal rotation $(\alpha)$ and of the octahedral flattening $(\psi)$ and are given in Table 1 of Takeda \& Ross (1995).

D $\quad D^{*}$

Upper $M_{O}$

Lower $M_{O}$

Upper $O_{B}$

Lower $O_{B}$
$(1 / 3,0,1 / 2)$

$(\overline{1} / 3,0, \overline{1} / 2)$

$[1 / 2-f(\alpha), 0,1 / 2+f(\psi)]$

$[1 / 2+f(\alpha), 0,1 / 2-f(\psi)]$
D*

$(\overline{1} / 3,0,1 / 2)$

$(1 / 3,0, \overline{1} / 2)$

$[1 / 2+f(\alpha), 0,1 / 2+f(\psi)]$

$[1 / 2-f(\alpha), 0,1 / 2-f(\psi)]$
$\mathrm{T}$

$(1 / 3,0,1 / 2)$

$(1 / 3,0, \overline{1} / 2)$

$[1 / 2+f(\alpha), 0,1 / 2+f(\psi)]$

$[1 / 2+f(\alpha), 0,1 / 2-f(\psi)]$
$\mathrm{T}^{*}$

$(\overline{1} / 3,0,1 / 2)$

$(\overline{1} / 3,0, \overline{1} / 2)$

$[1 / 2-f(\alpha), 0,1 / 2+f(\psi)]$

$[1 / 2-f(\alpha), 0,1 / 2-f(\psi)]$

Table 3. Relation between the integral hexagonal $L_{N}$ index referred to an $N$-layer cell and the real $L_{R}$ orthogonal reciprocal-lattice coordinate of the Fourier transform of the layer

$\begin{array}{lll} & \text { Orthohexagonal }(a, b) \text { axes } & \text { Hexagonal }\left(A_{1}, A_{2}\right) \text { axes } \\ \text { Orthogonal polytypes } & L_{R}=L_{N} / N & L_{R}=L_{N} / N \\ \text { Class a polytypes } & L_{R}=L_{N} / N+h / 3 N & L_{R}=L_{N} / N+H / 3 N \\ \text { Class } b \text { polytypes } & L_{R}=L_{N} / N+k / 3 N & L_{R}=L_{N} / N+(H+2 K) / 3 N\end{array}$

Pauling model the $O_{B}$ atoms also take the same ideal coordinates in all the four TS unit layers (Table 2). Besides, the contributions from the $M_{O}$ cations to the layer Fourier transform become identical when the following conditions are satisfied ( $c f$. Sadanaga \& Takeda, 1969; Takeda \& Sadanaga, 1969; Takeda \& Ross, 1995):

$$
\begin{gathered}
H=0(\bmod 3), \quad \text { all } K ; \\
H=1(\bmod 3), \quad K \neq 1(\bmod 3) ; \\
H=2(\bmod 3), \quad K \neq 2(\bmod 3) ; \\
h=0(\bmod 3), \quad \text { all } k ; \\
h \neq 0(\bmod 3), \quad k \neq 0(\bmod 3) .
\end{gathered}
$$

Consequently, $G_{j}$ is identical for all $j\left(G_{j}=G_{0}\right)$ and the contribution of the Fourier transform of the layer can be extracted from the summation in (3), obtaining the PID function $S^{N}$ :

$$
\begin{aligned}
S^{N}(H K . L) \cong & G^{N}(H K . L) / G_{0}\left(H K . L_{R}\right) \\
= & \sum_{j=1}^{N} \exp \left\{2 \pi i \left[H \Delta X_{j}+K \Delta Y_{j}\right.\right. \\
& +L(j-1) / N]\} .
\end{aligned}
$$

Since the non- $90^{\circ}$ angle can be either $\alpha$ or $\beta$, the relation between $L$ and $L_{R}$ depends on the Class and is given in Table 3 for orthohexagonal $(h, k)$ and hexagonal $(H, K)$ indices.

PID as defined in equation (5) exists in a subspace of the reciprocal space, which is identified by equation (4). As shown by Sadanaga \& Takeda (1969) (see their Fig. 4), (4) locates the subspace of the non-family reflections plus reciprocal-lattice rows parallel to $\mathbf{c}^{*}$ with both $h$ and $k=0(\bmod 3)$; these reflections, as stated above, are family reflections corresponding to the ninefold family structure (common to all mica polytypes).

Within the trigonal model, the $O_{B}$ atomic coordinates are no longer identical; however, from Table 2 and Fig. 3 it is easy to verify that the $O_{B}$ contribution to $G_{j}$ in all the four TS unit layers is the same when

$$
\begin{gathered}
H=0, \quad \text { all } K ; \quad K=0, \quad \text { all } H ; \quad H=-K ; \\
h=0, \quad \text { all } k ; \quad h= \pm k .
\end{gathered}
$$

For these reflections, $G_{j}=G_{0}$ and equation (5) holds again.

Finally, PID is defined in a subspace of the reciprocal space, which narrows from polytypes of subfamily A to mixed-rotation polytypes in the following way (Fig. 4).

(i) Polytypes of subfamily $\mathrm{A}$ involve only $2 n \times 60^{\circ}$ rotations of the $\mathrm{M}$ layer (same orientation parity of $\mathrm{Z}$ symbols; all-even RTW symbols) and are thus described by only one kind of TS layer (D or $\mathrm{D}^{*}$ depending on the orientation parity; see next paragraph). Therefore, equation (5) is valid in the whole reciprocal space. For identification of individual polytypes, PID calculated along each of the reciprocal-lattice rows parallel to $\mathbf{c}^{*}$ and belonging to non-family reflections can be used.

(ii) Polytypes of subfamily $\mathrm{B}$ involve only $(2 n+1) \times 60^{\circ}$ rotations of the $\mathrm{M}$ layer (alternating orientation parity of Z symbols; all-odd RTW symbols). For these polytypes, both $\mathrm{T}$ and $\mathrm{T}^{*}$ layers are necessary; in fact [equation (2)] $\mathrm{TT}^{*}$ and $\mathrm{TT}^{*}$ pairs are physically possible, whereas TT and $\mathrm{T}^{*} \mathrm{~T}^{*}$ pairs are not. In these two TS layers, the $x$ coordinates of $O_{B}$ atoms are identical (Table 2). Therefore, equation (5) is valid under the conditions given by (4). For identification purposes, PID in the whole subspace of the non-family reflections can be used also in this case. 
(iii) Mixed-rotation polytypes correspond to different non-alternating orientation parity of $\mathrm{Z}$ symbols, i.e. both even and odd RTW symbols; in general, they require all the four TS unit layers and equation (5) is valid only under the conditions given by (6). Therefore, PID is defined only for reciprocal-lattice rows parallel to $\mathbf{c}^{*}$ and belonging to the three planes $(0 k l),(h h l),(\bar{h} h l)$, which correspond to the first, third, fifth, eight, eleventh,... ellipses of the oblique-texture electron diffraction technique (Zvyagin, 1967).

In conclusion, PID is always defined at least in the three planes $(0 k l),(h h l)$ and $(\bar{h} h l)$.

\section{TS layers and choice of the axial setting}

The theory of axial settings recently introduced (Nespolo et al., 1998) aims at a unified geometrical treatment of the lattice of mica polytypes, mainly in view of dealing with the long-period polytypes, the stacking sequence of which can be determined by PID analysis. A crucial step in order to compute PID is the determination of the space orientation of the structural model in accordance with its space-group type. The correct procedure is analysed hereinafter in terms of Series, Classes and Subclasses, which represent the basis for the choice of the most suitable structure-related axial setting.

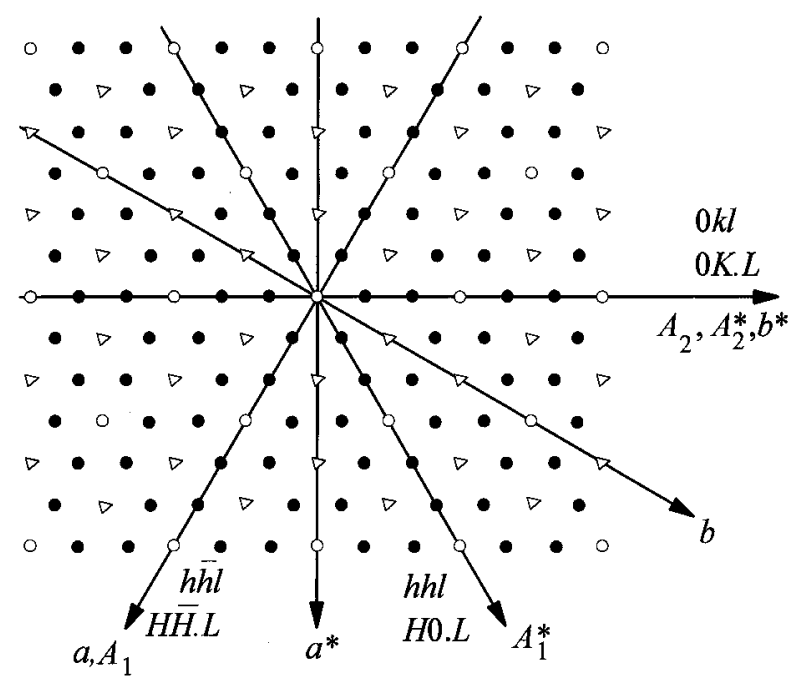

Fig. 4. (001) projection of mica reciprocal lattice. Direct- and reciprocal-lattice axes are shown, not on the same scale (hexagonal axes: $A_{1}, A_{2}, A_{1}^{*}, A_{2}^{*}$; orthohexagonal axes: $\left.a, b, a^{*}, b^{*}\right)$. The orthohexagonal axes locate the $C_{1}$ cell [ $c f$. Fig. 2 of Nespolo, Takeda \& Ferraris (1997)]. Open circles: family reflections for the ninefold family structure (common to all mica polytypes). Open triangles: family reflections for the threefold family structure but not for the ninefold one (different for subfamily A and subfamily B OD polytypes). Black circles: non-family reflections. The three planes indicated ( $h \mathrm{kl}$ : orthohexagonal indices; HK.L: hexagonal indices) are the only ones in which PID is defined in the case of mixedrotation polytypes [modified after Sadanaga \& Takeda (1969)].
In Series 0 [ $n=0$ in equation (1)], polytypes of subfamily A belong to Class a. By imposing $\mathbf{c}_{n}=(\overline{1} / 3,0)$, it is easy to show that Subclass 1 polytypes are described by all-odd $\mathrm{Z}$ symbols (D layers only), whereas Subclass 2 polytypes are described by all-even $\mathrm{Z}$ symbols ( $\mathrm{D}^{*}$ layers only). The origin of each layer is displaced by $-1 / 3$ (D layers) or $+1 / 3$ ( $D^{*}$ layers) along the $a$ axis and $0, \pm 1 / 3$ along the $b$ axis of the $C_{1}$ setting. The $c$ axis passes through the origin of each layer and in Subclass 1 it is displaced by $-a / 3$ for each stacking of layers; the resulting monoclinic angle is $\beta_{1 M}$. On the other hand, in Subclass 2, the $c$ axis is displaced $+a / 3$ for each stacking of layers and the resulting monoclinic angle is $\pi-\beta_{1 M}$, i.e. an acute monoclinic angle. For Subclass 2 polytypes, it is convenient to choose a monoclinic axial setting having $\mathbf{c}_{n}=(1 / 3,0)$, so that only the D layer is needed and the $c$ axis is again shifted by $-1 / 3$ along a for each stacking of layers, with a resulting monoclinic angle $\beta_{1 M}$. The correspondence between $\mathrm{Z}$ symbols and $\mathrm{D} / \mathrm{D}^{*}$ layers is, however, inverted, since $\mathrm{Z}$ symbols are defined with respect the $C_{1}$ setting and thus do not change. Taking the translationally reduced total displacement along the $-a$ semi-axis for Subclass 1 polytypes and along the $+a$ semi-axis for Subclass 2 polytypes, all Series 0 subfamily A polytypes can be described by only D layers, with (ideally) the same obtuse monoclinic angle of the $1 M$ polytype. The axial setting for Series 0 subfamily A polytypes corresponds thus to the Fixed-angle setting $\left({ }^{a} \mathbf{F}\right)$, introduced by Nespolo et al. (1998); for all polytypes belonging to a given Series, this setting has (ideally) the same monoclinic angle of the first (i.e. shortest period) polytype of that Series. Therefore, for Series 0 subfamily A polytypes, the simplest layer description (by means of $\mathrm{D}$ layers only) corresponds to the choice of the ${ }^{a} \mathbf{F}$ axial setting.

In the case of a higher Series, polytypes of subfamily A are either orthogonal or belong to Class $b$; in the second case, the simple relation between the Subclass and the parity of $\mathrm{Z}$ symbols (and thus the kind of TS layer), seen in the case of Series 0 , is no longer valid. In fact, in Class $b$ the parity of $\mathrm{Z}$ symbols depends on the relative proportion of 0,2 and -2 digits in the RTW symbols, and on their sequence. Besides, for subfamily B and for mixed-rotation polytypes, two and more than two different TS unit layers are necessary, respectively, and the displacement of each layer is not always in the same direction. The choice of the Fixed-angle axial settings ${ }^{\left(3^{n}, a ; 3^{n}, b\right)} \mathbf{F}$ (Nespolo et al., 1998) generalizes the criterion valid for Series 0 subfamily A polytypes and is the most natural setting when calculating PID by means of TS layers. Furthermore, for an $N$-layer polytype, the PID function $S^{N}\left(l_{N}\right)$ is periodic with $l_{N}=0, N-1$, where $l_{N}$ is the diffraction index referred to the $N$-layer unit cell. Since the origin of $S^{N}\left(l_{N}\right)$ is not fixed a priori, when comparing the computed and observed PID values, in principle the cyclic permutations of the $N$ 
Table 4. Transformation rules for $Z$ symbol sequences under the effect of the symmetry operators of the hexagonal syngony

$\mathrm{IJ} \ldots \mathrm{P}$ is the original sequence of $\mathrm{Z}$ symbols. Proper and improper motions have representation matrices with determinants +1 and -1 , respectively. Indexing is in orthohexagonal axes.

$\begin{array}{ll}\text { Proper motion } & \text { Effect on Z-symbol sequence } \\ {[001]_{2 n \pi / 6}} & n+\mathrm{I}, n+\mathrm{J}, \ldots,+n+\mathrm{P} \\ {[100]_{\pi}} & 3-\mathrm{P}, \ldots, 3-\mathrm{J}, 3-\mathrm{I} \\ {[310]_{\pi}} & 4-\mathrm{P}, \ldots, 4-\mathrm{J}, 4-\mathrm{I} \\ {[110]_{\pi}} & 5-\mathrm{P}, \ldots, 5-\mathrm{J}, 5-\mathrm{I} \\ {[3 \overline{1} 0]_{\pi}} & 8-\mathrm{P}, \ldots, 8-\mathrm{J}, 8-\mathrm{I} \\ {[1 \overline{1} 0]_{\pi}} & 7-\mathrm{P}, \ldots, 7-\mathrm{J}, 7-\mathrm{I} \\ {[010]_{\pi}} & 6-\mathrm{P}, \ldots, 6-\mathrm{J}, 6-\mathrm{I}\end{array}$

values should be tried, as well as the inverse sequence. In the ${ }^{\left(3^{n}, a ; 3^{n}, b\right)} \mathbf{F}$ axial setting, the origin is automatically fixed and that necessity is thus removed. However, special attention is needed in the case of triclinic polytypes belonging to Class $b$ and Series higher than 0 (see $\S 8)$.

\section{Structural model orientation and symbol conversion}

The six possible orientations of the structural model of each polytype with respect to the $(a, b)$ axes correspond to one and the same RTW symbol, but to six different sequences of both $\mathrm{Z}$ symbols and TS symbols, and are in general non-equivalent. Taking into account that:

(a) $\mathbf{c}_{n}$, translationally reduced, assumes components $(0,0)$ (orthogonal polytypes), $( \pm 1 / 3,[0, \pm 1 / 3])$ (Class a polytypes) and $(0, \pm 1 / 3)$ (Class $b$ polytypes);

(b) by rotation around $\mathbf{c}^{*}, \mathbf{c}_{n}$ for Class a polytypes can always be reduced to $( \pm 1 / 3,0)$ (Zvyagin, 1962, 1997); the six orientations of the structural model can be grouped in the following way.

(a) Class a polytypes. Each orientation corresponds to a different $\mathbf{c}_{n}$ projection.

(b) Class b polytypes. Three orientations correspond to $\mathbf{c}_{n}=(0,1 / 3)$ and three others to $\mathbf{c}_{n}=(0, \overline{1} / 3)$. The three orientations with the same $\mathbf{c}_{n}$ components are equivalent in the case of triclinic polytypes, but not for monoclinic ones. The symmetry elements in the $C_{1}$ setting must be disposed according to an $a$-unique setting. Only one of the three orientations leading to $\mathbf{c}_{n}=(0, \overline{1} / 3)$ agrees with such a requirement.

(c) Orthogonal polytypes. All the six orientations correspond to $\mathbf{c}_{n}=(0,0)$ and they are equivalent for hexagonal, trigonal and triclinic polytypes, while for orthorhombic and monoclinic polytypes only two orientations, related by a $180^{\circ}$ rotation around the $c=c^{*}$ axis, lead to the correct disposition of the symmetry elements.

The PID computation procedure described by Takeda \& Ross (1995) takes into account only the numerical values of $\mathbf{c}_{n}$ and not the equivalence/non-equivalence relations. It is thus incomplete for Class $b$ and orthogonal polytypes. By redefining the stacking operators $r_{j}$
Improper motion

(001)

(100)

(110)

(130)

(11̄0)

$(1 \overline{3} 0)$

(010)
Effect on Z-symbol sequen
$3+\mathrm{P}, \ldots, 3+\mathrm{J}, 3+\mathrm{I}$
$3-\mathrm{I}, 3-\mathrm{J}, \ldots, 3-\mathrm{P}$
$4-\mathrm{I}, 4-\mathrm{J}, \ldots, 4-\mathrm{P}$
$5-\mathrm{I}, 5-\mathrm{J}, \ldots, 5-\mathrm{P}$
$8-\mathrm{I}, 8-\mathrm{J}, \ldots, 8-\mathrm{P}$
$7-\mathrm{I}, 7-\mathrm{J}, \ldots, 7-\mathrm{P}$
$6-\mathrm{I}, 6-\mathrm{J}, \ldots, 6-\mathrm{P}$
$\mathrm{P}, \ldots, \mathrm{JI}$

introduced by Takeda \& Ross (1995) in terms of Z symbols, the correct structural model orientation can be obtained by analysing the symmetry properties of these symbols. Then, the calculation of the PID function requires the following steps.

Step 1 is the conversion from RTW symbols to 'provisional'_ Z symbols, by simply looking for $\mathbf{c}_{n}=(0,0),(\overline{1} / 3,0)$ or $(0, \overline{1} / 3)$. This is straightforwardly obtained by means of a simple addition cycle:

$$
Z_{j}=Z_{j-1}+A_{j-1} \quad(j=2-N) .
$$

$Z_{1}=3$ is assumed at first; if the resulting $\mathbf{c}_{n}$ projection does not take one of the three expected values, $Z_{1}$ is incremented and equation (7) is recalculated.

Step 2 is the derivation of the correct $\mathrm{Z}$ symbols, by analysing their symmetry properties. Symbols calculated by means of equation (7) display the full symmetry of the polytype. However, for orthogonal and Class $b$ polytypes, they may correspond to an orientation of the symmetry elements not compatible with the space-group type. In such a case, the sequence of $Z$ symbols must be changed, by making $Z_{1}$ take one of the other values leading to the same $\mathbf{c}_{n}$. This is tantamount to rotating the structural model around $\mathbf{c}^{*}$. The space-group type needs to be checked among only the 24 possible ones (Takeda, 1971) [for the relation between the two-dimensional pseudo-hexagonal and hexagonal lattices in the plane of the layer, see Nespolo, Takeda \& Ferraris (1997) and Nespolo, Takeda, Ferraris \& Kogure (1997)]. The symmetry properties of $\mathrm{Z}$ symbols for micas can be numerically expressed as in Table 4 , where the original sequence of $\mathrm{Z}$ symbols is assumed to be IJ ...P. The correct sequence is found when $Z$ symbols are related by symmetry operators located along the lattice directions compatible with the requirements of the space-group type.

Step 3 is the expression of the stacking operators $r_{j}$ (Takeda \& Ross, 1995) as a function of $\mathrm{Z}$ symbols and calculation of TS symbols. The stacking operators $r_{j}$ are here redefined as giving the displacement between the $(j-1)$ th and the $j$ th TS layers. Their $(a, b)$ components are indicated as $\left(x_{r_{i}}, y_{r_{j}}\right)$. The relation of the stacking operators $r_{j}$ with $\mathrm{Z}$ symbols is straightforward 
Table 5. Expression of the kind of TS unit layer $L_{j}$ in terms of RTW symbols $A_{j}$ and stacking operators $r_{j}$

\begin{tabular}{lll} 
& $r_{j}$ \\
\cline { 2 - 3 }$A_{j}$ & $0(\bmod 2)$ & $1(\bmod 2)$ \\
$0(\bmod 2)$ & $L_{j}=\mathrm{D}^{*}$ & $L_{j}=\mathrm{D}$ \\
$1(\bmod 2)$ & $L_{j}=\mathrm{T}^{*}$ & $L_{j}=\mathrm{T}$
\end{tabular}

for orthogonal polytypes, while for non-orthogonal polytypes the Subclass has to be taken into account. $\mathrm{Z}$ symbols for non-orthogonal polytypes always correspond to $(\overline{1} / 3,0)($ Class $a)$ or $(0, \overline{1} / 3)($ Class $b)$. PID is most conveniently expressed in the ${ }^{\left(3^{n}, a ; 3^{n}, b\right)} \mathbf{F}$ axial setting, which corresponds to $\mathbf{c}_{n}=(\overline{1} / 3,0)$ or $(0, \overline{1} / 3)$ for Subclass 1 and $\mathbf{c}_{n}=(1 / 3,0)$ or $(0,1 / 3)$ for Subclass 2. It follows that for orthogonal polytypes and Subclass 1 polytypes the stacking operators simply coincide with $\mathrm{Z}$ symbols $\left(r_{j}=Z_{j}\right)$, while for Subclass 2 polytypes they are related by a $180^{\circ}$ rotation around $\mathbf{c}^{*}\left(r_{j}=Z_{j}+3\right)$.

Step 4 is the computation of the PID $\left(S^{N}\right)$ as a function of $(a, b)$ components of TS symbols. The components of the $j$ th TS layer referred to the $(a, b)$ axes are indicated as $\left(X_{j}, Y_{j}\right)$, to distinguish them from the components in the $\left(A_{1}, A_{2}\right)$ axes, which were labelled $\left(\Delta X_{j}, \Delta Y_{j}\right)$ [equations (3) and (5)]. $\left(X_{j}, Y_{j}\right)$ are just the sum of the $\left(x_{r_{i}}, y_{r_{j}}\right)$ components from the first to $j$ th stacking operators. However, since the $c$ axis of the $\left(3^{n}, a ; 3^{n}, b\right) \mathbf{F}$ axial setting is displaced by $-1 / 3^{(n+1)}$ (where $n$ is the Series) along $a$ or $b$ (depending upon the Class), the additional displacement $\left[-j / 3^{(n+1)}, 0\right]($ Class $a)$ or $\left[0,-j / 3^{(n+1)}\right]\left(\right.$ Class b) must be added to the $\left(X_{j}, Y_{j}\right)$ component of the $j$ th TS symbol to express the layer stacking of non-orthogonal polytypes with respect to the $\left(3^{n}, a ; 3^{n}, b\right) \mathbf{F}$ axial setting. In this way, TS symbols for Series 0 subfamily A polytypes always have $X_{j}=0$ ( $c$ axis passing through the origin of each layer).

Orthogonal polytypes:

$$
\left(X_{j}, Y_{j}\right)=\sum_{i=1}^{j}\left(x_{r_{i}}, y_{r_{i}}\right)
$$

Class a polytypes:

$$
\left(X_{j}, Y_{j}\right)=\sum_{i=1}^{j}\left(x_{r_{i}}, y_{r_{i}}\right)+\left(-i / 3^{n+1}, 0\right)
$$

Class $b$ polytypes:

$$
\left(X_{j}, Y_{j}\right)=\sum_{i=1}^{j}\left(x_{r_{i}}, y_{r_{i}}\right)+\left(0,-i / 3^{n+1}\right) .
$$

Finally, in Class $b$ the axes exchange $a \leftrightarrow b$ has to be accomplished in order to obtain the $3^{3^{n}, b} \mathbf{F}$ axial setting. The complete TS symbols $L_{j}\left(X_{j}, Y_{j}\right)$ are easily obtained from Table 5 and (8), and the PID function $S^{N}$ is given in equation (9) [after Takeda \& Sadanaga (1969), notation slightly modified].

$$
\begin{aligned}
S^{N}(h k \hat{l})= & \sum_{j=1}^{N} S_{j}^{N}(h k \hat{l}) \\
= & \sum_{j=1}^{N} \exp \left\{2 \pi i\left[h X_{j}+k Y_{j}+\hat{l}(j-1) / N\right]\right\} \\
& {[\hat{l}=l(\bmod N)] }
\end{aligned}
$$

with the normalizing condition

$$
\sum_{j=1}^{N}\left[S_{j}^{N}(h k \hat{l})\right]^{2}=N^{2}
$$

It should be considered that equation (5) is based on the approximation of a trigonal distribution of each kind of atom in the layer and $G_{0}$ is thus an approximation of the Fourier transform of the layer. In the regions of the reciprocal space where $G_{0}$ passes through zero and changes sign, the relative error becomes large and equation (5) is actually no longer applicable. In the practice of mica polytype identification, the periods corresponding to $l$ intervals including those regions should not be used to derive the PID from measured intensities. These intervals depend on the actual chemical composition.

\section{Symmetry of PID}

The PID function in equation (9) can be rewritten as

$$
S^{N}(h k \hat{l})=\sum_{j=1}^{N} \exp \left(2 \pi i \varphi_{j}\right) \exp \{2 \pi i[\hat{l}(j-1) / N]\}
$$

where

$$
\varphi_{j}=h X_{j}+k Y_{j}
$$

Similarly,

$$
\begin{aligned}
S^{N}(2 h, 2 k, N-\hat{l})= & \sum_{j=1}^{N} \exp \left(2 \pi i 2 \varphi_{j}\right) \exp \{2 \pi i \\
& \times[(N-\hat{l})(j-1) / N]\} .
\end{aligned}
$$

For Series 0 polytypes, $\varphi_{j}$ can assume only the three values $0,1 / 3$ or $1 / 3$; it follows that:

$$
\left\{\begin{aligned}
\cos \left(2 \pi 2 \varphi_{j}\right) & =\cos \left(2 \pi \varphi_{j}\right)=\cos \left[2 \pi\left(-\varphi_{j}\right)\right] \\
\sin \left(2 \pi 2 \varphi_{j}\right) & =-\sin \left(2 \pi \varphi_{j}\right)=\sin \left[2 \pi\left(-\varphi_{j}\right)\right] .
\end{aligned}\right.
$$

Also,

$$
\left\{\begin{array}{l}
\cos \{2 \pi[(N-\hat{l})(j-1) / N]\}=\cos \{2 \pi[\hat{l}(j-1) / N]\} \\
\sin \{2 \pi[(N-\hat{l})(j-1) / N]\}=-\sin \{2 \pi[\hat{l}(j-1) / N]\}
\end{array}\right.
$$

and thus

$$
S^{N}(2 h, 2 k, \hat{l})=S^{N}(h, k, N-\hat{l}) .
$$

For Series 0 subfamily A polytypes, $\varphi_{j}$ in equation (11) simply becomes $k Y_{j}$ and, once more, it can assume only 
Table 6. Symbols, reflection conditions and PID values for subfamily A orthogonal polytype $\left(C_{1}\right.$ setting; hexagonal setting within parentheses)

$\begin{array}{lllllll}\text { Polytype } & \text { Space-group type } & \mathrm{RTW} & \mathrm{Z} & \mathrm{TS} & \text { Reflection conditions } & \mathrm{PID}(02 l) \\ 3 T & P 3_{1} 12 & {[2]_{3}} & 351 & \mathrm{D}(-1,0) \mathrm{D}(1,1) \mathrm{D}(0,0) & k=3 n: l=3 n+h & 1.7,1.7,1.7 \\ & P 3_{2} 12 & {[\overline{2}]_{3}} & 315 & \mathrm{D}(-1,0) \mathrm{D}(1,-1) \mathrm{D}(0,0) & {[H-K=3 n: L=3 n+H]} & 1.7,1.7,1.7\end{array}$

the three values $0,1 / 3$ or $-1 / 3$. From equations (12) and (14), it follows that

$$
S^{N}(0,2 k, \hat{l})=S^{N}(\stackrel{ \pm}{k} \hat{k} \hat{l})=S^{N}(\stackrel{ \pm}{k} k, N-\hat{l})
$$

For all polytypes belonging to Series 0 , the following relations hold [see equation (14)]:

$$
\begin{aligned}
& S^{N}(04 \hat{l})=S^{N}(02, N-\hat{l}) ; \\
& S^{N}(22 \hat{l})=S^{N}(11, N-\hat{l}) ; \\
& S^{N}(\overline{2} 2 \hat{l})=S^{N}(\overline{1} 1, N-\hat{l}) .
\end{aligned}
$$

Besides, in the case of OD polytypes, for which the PID is defined in the whole subspace of non-family reflections, since in Series $0, X_{j}$ and $Y_{j}$ may take only $0, \pm 1 / 3$, the following general relation holds:

$$
S^{N}(h+3 N, k+3 N, \hat{l})=S^{N}(h k \hat{l}) .
$$

Polytypes of subfamily A are described by only one kind of TS layer $\left(\mathrm{D}\right.$ or $\left.\mathrm{D}^{*}\right)$ and the component $X_{j}$ in equations (8) and (9) is always zero. Equation (16) shows that the distribution of the PID values has a trigonal symmetry, obeying the following relation:

$$
\begin{aligned}
S^{N}(02 \hat{l}) & =S^{N}(\overline{1} \hat{1} \hat{l})=S^{N}(1 \overline{1} \hat{l})=S^{N}(0 \overline{2}, N-\hat{l}) \\
& =S^{N}(11, N-\hat{l})=S^{N}(\overline{1} 1, N-\hat{l}) .
\end{aligned}
$$

Therefore, in Tables $6-14$, only $S^{N}(02 \hat{l})$ will be given for subfamily A Series 0 polytypes.

For higher Series, equations (17) and (18) are satisfied for orthogonal polytypes, but the length of the axis displacement for non-orthogonal polytypes is a submultiple of the layer stagger [see equation (8) and §8] and the same PID values correspond to different $l$ indices. With respect to equation (17), an origin shift and the inversion in the reading sequence appear, which depend in a complex way on $h$ and $k$. Subfamily A polytypes of Series $>0$ never belong to Class $a$; the $c$ axis is either perpendicular (orthogonal polytypes) or inclined towards $b$ (Class $b$ ); for a $3 N$-layer polytype, the $X_{j}$ components corresponding to $j=0,1,2(\bmod 3)$ take the sequences $(1 / 3, \overline{1} / 3,0)_{N}$ or $(\overline{1} / 3,1 / 3,0)_{N}$ depending on the orientation parity and, for Class $b$ polytypes, on the Subclass. Again, because of the fractional relation between axis displacement and the layer stagger, an origin shift occurs and equation (18) is no longer satisfied. Finally, in the case of subfamily B and mixed-rotation polytypes, more than one kind of TS unit layer is required and $X_{j}$ can take all the possible values in different sequences, depending on the polytype. As a consequence, equation (19) reduces to the centrosymmetrical relation $S^{N}(h k \hat{l})=S^{N}(\bar{h} \bar{k}, N-\hat{l})$.

\section{Reflection conditions}

In the diffraction patterns of mica polytypes, systematic absences are due either to the space-group type [general reflection conditions (Hahn \& Vos, 1996)] or to local symmetry [additional reflection conditions (Hahn \& Vos, 1996), Templeton effect (Templeton, 1956; Pabst, 1959, 1961; Rumanova \& Belov, 1961), non-characteristic orbits of space groups (Wondratschek, 1976; Matsumoto \& Wondratschek, 1979, 1987)]. These absences may affect part or all of the non-family reflections. The reflection conditions are part of the information contained in the PID function. PID is however defined only for a subspace of the non-family reflections, at least for mixed-rotation polytypes. For these polytypes, the reflection conditions appearing in the subspace where PID is not defined may convey some information useful for a preliminary analysis. The complete reflection conditions for mica polytypes can be obtained by Zvyagin's functions (Zvyagin, 1967; Nespolo \& Kogure, 1998), i.e. by a form of the structure factor parameterized versus the features of the polytypes. Since the number of polytypes grows very rapidly with the number of layers, in Tables 6-14 the complete reflection conditions for mica polytypes with up to four layers only are given.

There are 1,2, 6 and 26 possible mica polytypes with $1,2,3$ and 4 layers, respectively, listed by Ross et al. (1966). Tables 6-14 give space-group type, RTW, Z and TS symbols, calculated reflection conditions and computed PID values for these polytypes in the $C_{1}$ (orthogonal polytypes) or ${ }^{\left(3^{n}, a ; 3^{n}, b\right)} \mathbf{F}$ axial settings (nonorthogonal polytypes). Hexagonal indices for the $3 T$ polytype are written in capital letters. In the real structure, the additional reflection conditions correspond to low but nonzero values of the structure factor: this is especially true in the case of dioctahedral micas, where one of the octahedral sites is more distorted and (ideally) vacant, although not always completely (Pavese et al., 1997), and the corresponding weak reflections have been called 'dioctahedral reflections' (Rieder, 1968). 
Table 7. Symbols, reflection conditions and PID values for subfamily B orthogonal polytypes $\left(C_{1}\right.$ setting

$\begin{array}{llllll}\text { Polytype } & \text { Space-group type } & \mathrm{RTW} & \mathrm{Z} & \mathrm{TS} & \text { Reflection conditions } \\ 2 O & C c c m & {[3]_{2}} & 36 & \mathrm{~T}(-1,0) \mathrm{T}^{*}(0,0) & h=3 n: l=2 n \\ 4 M_{6} & C 2 / c & 11 \overline{1} 1 & 3432 & \mathrm{~T}(-1,0) \mathrm{T}^{*}(0,1) \mathrm{T}(-1,1) \mathrm{T}^{*}(0,0) & h=3 n, k=3 n: l=4 n \\ & & & & h=3 n, k \neq 3 n: l \neq 4 n+2 \\ & & & & h \neq 3 n, k=3 n: l=2 n\end{array}$

\begin{tabular}{lll} 
PID & & \\
\hline $02 l$ & $11 l$ & $-11 l$ \\
$2.0,0.0$ & $1.0,1.7$ & $1.0,1.7$ \\
$2.0,2.45,0.0,2.45$ & $1.0,3.3,1.7,0.9$ & $1.0,0.9,1.7,3.3$
\end{tabular}

Table 8. Symbols, reflection conditions and PID values for mixed-rotation orthogonal polytypes $\left(C_{1}\right.$ setting)

\begin{tabular}{|c|c|c|c|c|c|}
\hline Polytype & Space-group type & RTW & $\mathrm{Z}$ & TS & Reflection conditions \\
\hline $4 O_{1}$ & Ccmm & 0303 & 3366 & $\mathrm{D}(-1,0) \mathrm{T}(1,0) \mathrm{D}^{*}(-1,0) \mathrm{T}^{*}(0,0)$ & $h=3 n: l=4 n$ \\
\hline $4 \mathrm{O}_{2}$ & $C 2 \mathrm{~cm}$ & $13 \overline{1} 3$ & 1254 & $\mathrm{~T}(-1,-1) \mathrm{T}^{*}(0,1) \mathrm{T}(-1,-1) \mathrm{T} *(0,0)$ & $\begin{array}{l}h=3 n, k=3 n: l=4 n \\
h \neq 3 n, k=3 n: l=2 n\end{array}$ \\
\hline $4 O_{3}$ & $C c 2 m$ & $23 \overline{2} 3$ & 2415 & $\mathrm{D} *(1,-1) \mathrm{T} *(-1,0) \mathrm{D}(1,-1) \mathrm{T}(0,0)$ & $\begin{array}{l}h=3 n, k=3 n: l=4 n \\
h=3 n, k \neq 3 n: l=2 n\end{array}$ \\
\hline $4 O_{4}$ & $C 222_{1}$ & 1212 & 1245 & $\mathrm{~T}(-1,-1) \mathrm{D} *(0,1) \mathrm{T} *(1,-1) \mathrm{D}(0,0)$ & $\begin{array}{l}h=3 n, k=3 n: l=4 n \\
h \neq 3 n, k \neq 3 n: l \neq 4 n+2\end{array}$ \\
\hline $4 M_{7}$ & $C 2$ & $01 \overline{2} 1$ & 3342 & $\mathrm{D}(-1,0) \mathrm{T}(1,0) \mathrm{D} *(-1,1) \mathrm{T} *(0,0)$ & $h=3 n, k=3 n: l=4 n$ \\
\hline
\end{tabular}

\begin{tabular}{lll} 
PID & & \\
\hline $02 l$ & $11 l$ & $-11 l$ \\
$4.0,0.0,0.0,0.0$ & $1.0,1.7,3.0,1.7$ & $1.0,1.7,3.0,1.7$ \\
$1.0,1.7,3.0,1.7$ & $2.6,1.7,1.7,1.7$ & $2.6,1.7,1.7,1.7$ \\
$2.0,0.0,3.5,0.0$ & $2.6,1.7,1.7,1.7$ & $2.6,1.7,1.7,1.7$ \\
$1.0,1.7,3.0,1.7$ & $2.0,2.45,0.0,2.45$ & $2.0,2.45,0.0,2.45$ \\
$2.6,1.7,1.7,1.7$ & $1.0,3.3,1.7,0.9$ & $1.0,0.9,1.7,3.3$
\end{tabular}


Table 9. Symbols, reflection conditions and PID values for subfamily A Series 0 Class a polytypes ( ${ }^{a} \boldsymbol{F}$ setting)

\begin{tabular}{|c|c|c|c|c|c|c|}
\hline Polytype & Space-group type & RTW & $\mathrm{Z}$ & TS & Reflection conditions & PID (02l) \\
\hline $2 M_{1}$ & $C 2 / c$ & $2 \overline{2}$ & 24 & $\mathrm{D}(0,1) \mathrm{D}(0,0)$ & $k=3 n: l=2 n$ & $1.0,1.7$ \\
\hline $4 M_{1}$ & $C 2 / c$ & $020 \overline{2}$ & 5511 & $\begin{array}{l}\mathrm{D}(0,1) \mathrm{D}(0,-1) \\
\mathrm{D}(0,1) \mathrm{D}(0,0)\end{array}$ & $k=3 n: l=4 n$ & $1.0,1.7,3.0,1.7$ \\
\hline $4 M_{2}$ & $C 2$ & 0222 & 3351 & $\begin{array}{l}\mathrm{D}(0,0) \mathrm{D}(0,0) \\
\mathrm{D}(0,1) \mathrm{D}(0,0)\end{array}$ & Same as $4 M_{1}$ & $2.6,1.7,1.7,1.7$ \\
\hline $4 A_{1} \dagger$ & $C \overline{1}$ & $002 \overline{2}$ & 1113 & $\begin{array}{l}\mathrm{D}(0,-1) \mathrm{D}(0,1) \\
\mathrm{D}(0,0) \mathrm{D}(0,0)\end{array}$ & Same as $4 M_{1}$ & $1.0,0.9,1.7,3.3$ \\
\hline $4 M_{3}$ & $C 2 / c$ & $2 \overline{2} \overline{2} 2$ & 3531 & $\begin{array}{l}\mathrm{D}(0,0) \mathrm{D}(0,1) \\
\mathrm{D}(0,1) \mathrm{D}(0,0)\end{array}$ & $\begin{array}{l}k=3 n: l=4 n \\
k \neq 3 n: l \neq 4 n+2\end{array}$ & $2.0,2.45,0.0,2.45$ \\
\hline
\end{tabular}

$\dagger$ Labelled 4 Tc8 in Ross et al. (1966).

\section{Discussion}

The analysis of the PID of mica polytypes is a powerful method to solve the stacking sequence of these silicates. It can be applied to polytypes of very long period, which could not be solved or would require very complex solution strategies (e.g. Kogure \& Nespolo, 1999a). In spite of the fact that the difference between the PID of polytypes with only slightly different stacking sequences is small, Kogure \& Nespolo (1999a) have shown that this minimal difference was still sufficient to determine the stacking sequence of a well crystallized 36-layer polytype, i.e. the longest mica polytype reported to date. For long-period polytypes, the quality of the crystal might, in principle, become critical, but even in the worst cases PID analysis drastically reduces the number of stacking candidates.

Since PID is the Fourier transform of the stacking vectors giving the relative displacements between TS layers, it is defined in the homo-octahedral approximation. The presence of M2 layers is in principle revealed by a structure refinement.

The calculation of the PID function starting from RTW symbols is accomplished by a computer program (PTST98) written in Fortran V, which can be obtained upon request from the correspondence author. Application of the program has been exemplified by Kogure \& Nespolo (1999a).

\subsection{A preliminary analysis to the application of PID}

The analysis of the geometry of the diffraction pattern should precede the PID analysis, since it offers important preliminary information, which can be summarized as follows.

(i) An analysis of the diffraction-pattern geometry is of particular importance in the case of (suspected) polytypes belonging to Series $>0$, when twinning may be present (Nespolo, Takeda \& Ferraris, 1997; Nespolo, Takeda, Ferraris \& Kogure, 1997; Nespolo, 1999).

(ii) The number of reflections along reciprocal-lattice rows corresponding to family reflections of the threefold family structure unequivocally indicate if a mica poly- type is an OD subfamily A, OD subfamily B or mixedrotation polytype.

(iii) The possibility exists of finding the same PID for polytypes with a different lattice; this is the case for $2 \mathrm{O}$ $\left(C_{1}\right.$ setting $)$ and $2 M_{2}\left({ }^{b} \mathbf{F}\right.$ setting $)$ polytypes, which have identical TS symbols (and thus the same PID).

(iv) As shown by Nespolo (1999), only in the ${ }^{a} \mathbf{F}$ settings are the family reflections for Series 0 polytypes identical, while this is not true in other settings.

(v) Special attention has to be paid in the case of triclinic polytypes belonging to Class $b$, which admits three equivalent orientations, related by $2 n \times 60^{\circ}$ rotations around $\mathbf{c}^{*}$. $Z$ symbols and TS symbols are different for the three orientations but they describe three equivalent orientations of the structural model. PID values expressed for a given reciprocal-lattice row in a certain orientation of the structural model correspond to a different row in another orientation. Since these rows are related by $2 n \times 60^{\circ}$ rotations around $\mathbf{c}^{*}$, for Series 0 polytypes the corresponding PID values are in their turn related by equation (17) or (19). For Series $>0$ polytypes, the comparison requires more attention. In this case, the $c$ axis of the ${ }^{\left(3^{n}, a ; 3^{n}, b\right)} \mathbf{F}$ setting is displaced by $1 / 3^{n}$ for each layer and the length of the axis displacement is a submultiple of the layer stagger; therefore, the origin of the PID is not the same in the three orientations of the structural model. In Table 14, the example of the $3 A_{1}$ polytype is presented. The existence of a similar ambiguity in chlorite was reported by Brindley et al. (1950).

\subsection{Comparison with previous results}

Comparison of the general results of the present research with previous partial reports reveals some errors.

Ross et al. (1966) gave partial reflection conditions for polytypes with up to four layers (see their Table 2). However, the possible ambiguities of the axial setting choice were not recognized and different settings were used to index different polytypes (four different kinds of settings for $2 M_{1}, 3 M_{1}, 4 M_{9}, 4 A_{2}, 4 A_{9}$ and $4 A_{10}$ polytypes; 


$\begin{array}{lllll}\text { Polytype } & \text { Space-group type } & \mathrm{RTW} & \mathrm{Z} & \mathrm{TS} \\ 4 M_{4} & C 2 / m & 0033 & 6663 & \mathrm{D} *(-1,0) \mathrm{D}^{*}(1,0) \mathrm{T}^{*}(0,0) \mathrm{T}(0,0) \\ 4 M_{5} & C 2 & 1122 & 2346 & \mathrm{~T}(-1,-1) \mathrm{T}(-1,-1) \mathrm{D}(1,0) \mathrm{D}^{*}(0,0) \\ 4 A_{2} & C \overline{1} & 1 \overline{1} \overline{1} 2 & 6164 & \mathrm{~T} *(-1,0) \mathrm{T}(-1,-1) \mathrm{D}^{*}(1,-1) \mathrm{D}^{*}(0,0) \\ & & & & \\ 4 A_{3} & C 1 & 13 \overline{2} \overline{2} & 2364 & \mathrm{~T} *(-1,-1) \mathrm{T}(-1,-1) \mathrm{D}^{*}(1,-1) \mathrm{D}^{*}(0,0) \\ 4 A_{4} & C 1 & & & \\ 4 A_{5} & C 1 & 0213 & 2245 & \mathrm{D}^{*}(-1,-1) \mathrm{D}^{*}(1,1) \mathrm{T}^{*}(0,-1) \mathrm{T}(0,0) \\ 4 A_{11} & C 1 & 0132 & 6614 & \mathrm{D}^{*}(-1,0) \mathrm{T}^{*}(1,0) \mathrm{T}(1,-1) \mathrm{D}^{*}(0,0) \\ 4 A_{8} \dagger & C \overline{1} & 011 \overline{2} & 4456 & \mathrm{D}^{*}(-1,1) \mathrm{T}^{*}(1,-1) \mathrm{T}(1,0) \mathrm{D}^{*}(0,0) \\ & & 2 \overline{2} 33 & 2425 & \mathrm{D}^{*}(-1,-1) \mathrm{D}^{*}(1,0) \mathrm{T}^{*}(0,-1) \mathrm{T}(0,0) \\ 4 A_{9} & C \overline{1} & & & \\ & & 1 \overline{2} 2 \overline{1} & 1262 & \mathrm{~T}(0,-1) \mathrm{D}^{*}(-1,1) \mathrm{D}^{*}(1,1) \mathrm{T}^{*}(0,0) \\ 4 A_{10} & C \overline{1} & & & \end{array}$

Reflection conditions

\section{$h=3 n: l=4 n$}

$h=3 n, k=3 n: l=4 n$

$h=3 n, k \neq 3 n: l \neq 4 n+2$

$h=3 n, k=3 n: l=4 n$

$h=3 n, k \neq 3 n: l \neq 4 n+2$

$h \neq 3 n, k=3 n-h: l=2 n$

$h=3 n, k=3 n: l=4 n$

$h(\bmod 3)=k(\bmod 3) \neq 3 n: l \neq 4 n+2$

$h=3 n, k=3 n: l=4 n$

Same as $4 A_{4}$

Same as $4 A_{4}$

$h=3 n, k=3 n: l=4 n$

$h \neq 3 n, k=3 n-h: l \neq 4 n+2$

$h=3 n, k=3 n: l=4 n$

$h(\bmod 3)=k(\bmod 3) \neq 3 n: l=2 n$

$h \neq 3 n, k=3 n-h: l \neq 4 n+2$

$h+k=3 n: l=4 n$ $h=3 n, k \neq 3 n: l=2 n$

PID

\begin{tabular}{lll}
\hline $02 l$ & $11 l$ & $-11 l$ \\
$4.0,0.0,0.0,0.0$ & $1.0,3.3,1.7,0.9$ & $1.0,0.9,1.7,3.3$ \\
$2.0,2.45,0.0,2.45$ & $2.6,1.7,1.7,1.7$ & $2.6,1.7,1.7,1.7$ \\
& & \\
$2.0,2.45,0.0,2.45$ & $1.0,3.3,1.7,0.9$ & $2.0,0.0,3.5,0.0$ \\
& & \\
$2.6,1.7,1.7,1.7$ & $2.0,2.45,0.0,2.45$ & $2.6,1.7,1.7,1.7$ \\
& & \\
$1.0,1.7,3.0,1.7$ & $1.0,0.9,1.7,3.3$ & $2.6,1.7,1.7,1.7$ \\
$2.6,1.7,1.7,1.7$ & $1.0,3.3,1.7,0.9$ & $1.0,1.7,3.0,1.7$ \\
$1.0,3.3,1.7,0.9$ & $2.6,1.7,1.7,1.7$ & $1.0,1.7,3.0,1.7$ \\
$2.0,0.0,3.5,0.0$ & $1.0,0.9,1.7,3.3$ & $2.0,2.45,0.0,2.45$ \\
& & \\
$1.0,0.9,1.7,3.3$ & $2.0,0.0,3.5,0.0$ & $2.0,2.45,0.0,2.45$ \\
& & \\
$1.0,0.9,1.7,3.3$ & $1.0,0.9,1.7,3.3$ & $4.0,0.0,0.0,0.0$
\end{tabular}

$\dagger$ Labelled $4 T c_{1}$ in Ross et al. (1966).

Table 11. Symbols, reflection conditions and PID values for subfamily B Series 0 Class b polytypes ( ${ }^{b} \boldsymbol{F}$ setting)

\begin{tabular}{|c|c|c|c|c|c|c|c|c|}
\hline \multirow[b]{2}{*}{ Polytype } & \multirow[b]{2}{*}{ Space-group type } & \multirow[b]{2}{*}{ RTW } & \multirow[b]{2}{*}{$\mathrm{Z}$} & \multirow[b]{2}{*}{ TS } & \multirow[b]{2}{*}{ Reflection conditions } & \multicolumn{3}{|l|}{ PID } \\
\hline & & & & & & $20 l$ & $11 l$ & $-11 l$ \\
\hline $2 M_{2}$ & $C 2 / c$ & $1 \overline{1}$ & 45 & $\mathrm{~T}(-1,0) \mathrm{T}^{*}(0,0)$ & $k=3 n: l=2 n$ & $2.0,0.0$ & $1.0,1.7$ & $1.0,1.7$ \\
\hline $4 M_{9}$ & $C 2$ & 1131 & 4563 & $\mathrm{~T} *(1,-1) \mathrm{T}(0,1) \mathrm{T} *(1,-1) \mathrm{T}(0,0)$ & $\begin{array}{l}h=3 n, k=3 n: l=4 n \\
h=3 n, k \neq 3 n: l=2 n\end{array}$ & $1.0,1.7,3.0,1.7$ & $2.6,1.7,1.7,1.7$ & $2.6,1.7,1.7,1.7$ \\
\hline $4 A_{6}$ & $C \overline{1}$ & $1 \overline{1} 33$ & 4541 & $\mathrm{~T}^{*}(1,-1) \mathrm{T}(0,1) \mathrm{T}^{*}(1,0) \mathrm{T}(0,0)$ & $\begin{array}{l}h=3 n, k=3 n: l=4 n \\
h=3 n, k \neq 3 n: l=2 n \\
h(\bmod 3)=k(\bmod 3) \neq 3 n: l \neq 4 n+2\end{array}$ & $1.0,0.9,1.7,3.3$ & $2.0,2.45,0.0,2.45$ & $1.0,3.3,1.7,0.9$ \\
\hline
\end{tabular}




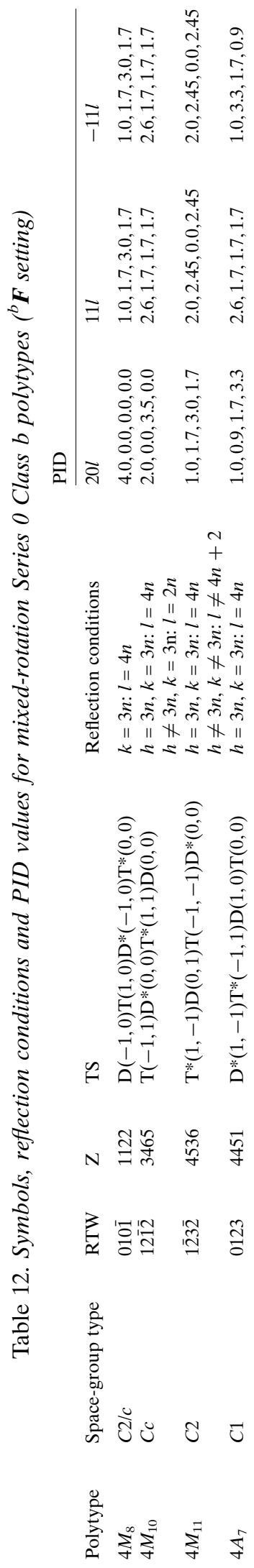

for $3 A_{2}$ polytype; for $4 A_{6}$ polytype; and for $4 M_{8}$ polytype) or different planes of the same polytype $\left(3 A_{1}, 4 M_{1}\right.$, $4 M_{2}, 4 M_{3}$ and $4 A_{8}$ ). Besides, as shown by Nespolo \& Kogure (1998), for the $3 T$ polytype a monoclinic setting was chosen, having an inclined $a^{*}$ axis parallel to that of the $2 M_{1}$ polytype. Finally, the partial reflection conditions given for $4 O_{2}, 4 M_{6}, 4 M_{9}, 4 A_{2}, 4 A_{6}, 4 A_{8}$ and $4 A_{9}$ are correct only for $h$ and/or $k \neq 0(\bmod 3)$. The reflection conditions analysed for one or two planes only and not for the whole reciprocal lattice erroneously led to $4 M_{1}$, $4 M_{2}$ and $4 M_{3}$ being considered as indistinguishable. Actually, the last one can be differentiated from the previous two by means of the reflection conditions in the ( $h h l)$ plane, as shown by Zhukhlistov et al. $(1988,1990) . \dagger$ The same remark can be made for $4 A_{3}, 4 A_{4}$ and $4 A_{5}$ polytypes: the first one can be distinguished from the other two by looking at the geometry of the $(h h l)$ plane (Tables 9 and 10).

Takeda \& Ross (1995) gave the reflection conditions that are part of the PID information for the 26 4-layer polytypes (see their Table 6) and the computed PID values for several subfamily A polytypes belonging to the three $1 M, 2 M_{1}$ and $3 T$ structural series. $\$$ However, since the structural model orientation was not taken into account, a few mistakes appeared in their Table 6 . The reflection conditions for $4 O_{3}$ and $4 M_{8}$ polytypes are given for $(h h l)-(\bar{h} h l)$ planes instead of for the $(0 \mathrm{kl})$ plane. The reflection conditions for the $4 O_{4}$ polytype in the $(h h l)-(\bar{h} h l)$ planes have not been given, while those in the $(0 \mathrm{kl})$ plane of the $4 M_{9}$ polytype should not be present. The space-group type of the $4 M_{10}$ polytype has been misprinted as $C 2 / c$ instead of $C c$ [the same misprint occurs in the papers by Ross et al. (1966) and Takeda (1971)]. Another misprint is in their Table 4, where the third value of the PID for the $(h h l)-(\bar{h} h l)$ planes of the $3 M_{2}$ polytype is 2.5 and not 0.9 . On the basis of the generalized notation introduced by Nespolo et al. (1998), all the axial settings labelled $1 M$ should be changed into ${ }^{a} \mathbf{F}$, and those labelled $3 M_{1}$ into ${ }^{3, a} \mathbf{F}$; the settings $3 T c_{1}$ and $9 T c_{1}$ become ${ }^{3, b} \mathbf{F}$ and ${ }^{9, b} \mathbf{F}$ if the axial exchange $a \leftrightarrow b$ is accomplished. All polytypes belonging to structural series $3 T$ listed in Table 9 of Takeda \& Ross (1995) belong to Class $a$ and the axial setting in which PID is expressed is ${ }^{a} \mathbf{F}$.

Polytype $3 A_{1}$ belongs to subfamily A but it is a Series 1 Class $b$ polytype. It is described by $\mathrm{M}$ layers in even orientation but, being a triclinic polytype, three orientations of the structural model are possible. Among

$\dagger$ The orthohexagonal 12-layer indexing given by Zhukhlistov et al. $(1988,1990)$ corresponds to the 4-layer monoclinic indexing shown in Table 9 of the present paper if the condition of integrality of monoclinic indices is taken into account.

¥ The definition of 'series' in Takeda \& Ross (1995) corresponds to that of 'structural series' in Baronnet (1978): it indicates the shortperiod polytype that is thought to have given origin to the long-period ones. This should not be confused with the definition of Series by Nespolo et al. (1998), which has a geometrical meaning. 
Table 13. Symbols, reflection conditions and PID values for mixed-rotation Series 1 Class a non-orthogonal polytypes $\left({ }^{a} \boldsymbol{S} \equiv{ }^{3 a} \boldsymbol{F}\right.$ setting)

\begin{tabular}{|c|c|c|c|c|c|c|c|c|}
\hline \multirow[b]{2}{*}{ Polytype } & \multirow[b]{2}{*}{$\begin{array}{l}\text { Space-group } \\
\text { type }\end{array}$} & \multirow[b]{2}{*}{ RTW } & \multirow[b]{2}{*}{$\mathrm{Z}$} & \multirow[b]{2}{*}{ TS } & \multirow[b]{2}{*}{ Reflection conditions } & \multicolumn{3}{|l|}{ PID } \\
\hline & & & & & & $02 l$ & $11 l$ & $-11 l$ \\
\hline $3 M_{1}$ & $C 2 / m$ & 033 & 336 & $\mathrm{D}(-2,0) \mathrm{T}(-4,0) \mathrm{T} *(0,0)$ & $h=3 n: l=3 n-h / 3$ & $3.0,0.0,0.0$ & $1.3,2.5,0.9$ & $1.3,0.9,2.5$ \\
\hline $3 M_{2}$ & $C 2$ & $11 \overline{2}$ & 561 & $\mathrm{~T}(-2,1) \mathrm{T}^{*}(2,1) \mathrm{D}(0,0)$ & $h=3 n, k=3 n: l=3 n-h / 3$ & $1.7,1.7,1.7$ & $0.9,1.3,2.5$ & $0.9,2.5,1.3$ \\
\hline $3 A_{2}$ & $C \overline{1}$ & $01 \overline{1}$ & 112 & $\mathrm{D}(-2,-1) \mathrm{T}(-4,1) \mathrm{T} *(0,0)$ & $h=3 n: l=3 n+k-h / 3$ & $0.0,0.0,3.0$ & $0.9,1.3,2.5$ & $2.5,1.3,0.9$ \\
\hline $3 A_{3}$ & $C 1$ & 123 & 235 & $\mathrm{~T} *(4,-1) \mathrm{D}(2,-1) \mathrm{T}(0,0)$ & Same as $3 M_{2}$ & $1.7,1.7,1.7$ & $2.5,0.9,1.3$ & $1.3,0.9,2.5$ \\
\hline
\end{tabular}

Table 14. Symbols, reflection conditions and PID values for subfamily A Series 1 Class b non-orthogonal polytype $\left({ }^{b} \boldsymbol{S} \equiv{ }^{3 b} \boldsymbol{F}\right.$ setting)

\begin{tabular}{lllllllll} 
& Space-group & & & & & PID & \\
\cline { 5 - 9 } Polytype & type & RTW & $\mathrm{Z}$ & $\mathrm{TS}$ & Reflection conditions & $20 l$ & $11 l$ \\
$3 A_{1}$ & $C \overline{1}$ & $02 \overline{2}$ & 662 & $\mathrm{D}^{*}(1,1) \mathrm{D}^{*}(-1,2) \mathrm{D}^{*}(0,0)$ & $h=3 n: l=3 n-h / 3-k$ & $1.3,2.5,0.9$ & $0.9,2.5,1.3$ & $1.3,2.5,0.9$ \\
& & & 224 & $\mathrm{D}^{*}(1,-2) \mathrm{D}^{*}(-1,-4) \mathrm{D}^{*}(0,0)$ & & $0.9,1.3,2.5$ & $2.5,1.3,0.9$ & $0.9,1.3,2.5$ \\
& & & 446 & $\mathrm{D}^{*}(1,4) \mathrm{D}^{*}(-1,-1) \mathrm{D}^{*}(0,0)$ & & $2.5,0.9,1.3$ & $1.3,0.9,2.5$ & $2.5,0.9,1.3$
\end{tabular}

these, one corresponds to $\mathrm{Z}$ symbols 2 and 4 , as polytype $2 M_{1}$. The period along $\mathbf{c}^{*}$ of an $N$-layer polytype is $c_{N}^{*}=N c_{1}^{*}$ (Nespolo et al., 1998). The appearance of $(h 0 l)$ and $(h .3 h . l)$ planes $\left(C_{1}\right.$ setting), containing only family reflections, is the same for $2 M_{1}$ and $3 A_{1}$ polytypes (Borutskiy et al., 1987) $\dagger$ However, this identity does not hold for other planes, where non-family reflections appear and no common axial setting can be found, since polytypes belonging to a Series cannot be indexed in the monoclinic setting of any polytype belonging to another Series.

We are deeply indebted to Professor Boris B. Zvyagin (IGEM, RAS, Moscow), Professor Slavomil Ďurovič (Slovak Academy of Sciences, Bratislava) and Professor Jillian F. Banfield (University of Wisconsin-Madison) for several fundamental discussions and for critical reading of the manuscript. We thank Professor Yoshio Takéuchi (Nihon University, Tokyo), Professor Ryoichi Sadanaga (Japan Academy), Dr Malcom Ross (US Geological Service), Professor Stefano Merlino (University of Pisa), Professor Takeo Matsumoto (University of Kanazawa) and Dr Akiji Yamamoto (NIRIM, Tsukuba) for valuable discussions, and Professor D. Yu. Pushcharovsky (Moscow State University) for sending consultation material. MN is also indebted to Professor Masamichi Miyamoto (University of Tokyo) and Professor Yasunari Watanabe (Teikyo Heisei University) for their constant encouragement. This research has been partly developed during a Doctoral Program (MN) supported by the Japanese Ministry of Education, Science and Culture.

$\dagger$ Borutskiy et al. (1987) gave odd $\mathrm{Z}$ symbols for the $3 A_{1}$ polytype, namely 113 , while it must be described by even $\mathrm{Z}$ symbols.
GF acknowledges financial support from the Italian National Research Council (CNR) and from the Italian Ministry of University and of Scientific and Technological Research (MURST).

\section{References}

Andreeva, N. S. (1962). Sov. Phys. Crystallogr. 6, 419-422.

Arnold, H. (1996). International Tables for Crystallography, Vol. A, edited by Theo Hahn, part 5. Dordrecht: Kluwer Academic Publishers.

Backhaus, K.-O. (1983). Cryst. Res. Techn. 18, 1253-1260.

Backhaus, K.-O. \& Ďurovič, S. (1984). Clays Clay Miner. 32, 453-463.

Bailey, S. W. \& Christie, O. H. J. (1978). Am. Mineral. 63, 203-204.

Baronnet, A. (1978). Progress in Crystal Growth and Characteristics, Vol. 1, pp. 151-211. Oxford: Pergamon Press.

Belov, N. V. (1949). Mineralog. Sb. L'vovsk. Geol. Obshchestvo pri L'vovsk. Gos. Univ. 3, 29-40. (In Russian.)

Borutskiy, B. Y., Soboleva, S. V. \& Golovanova, T. I. (1987). Dokl. Acad. Sci. USSR Earth Sci. Sect. 294, 141-143.

Brindley, G. W., Oughton, B. M. \& Robinson, K. (1950). Acta Cryst. 3, 408-416.

Catti, M., Ferraris, G. \& Ivaldi, G. (1989). Eur. J. Miner. 1, 625-632.

Černý, P., Rieder, M. \& Povondra, P. (1970). Lithos, 3, 319-325.

Dekeyser, W. \& Amelinckx, S. (1953). C. R. XIX Session Congr. Geol. Intern. Comité International pour l'Étude des Argiles, Alger, fasc. XVIII, pp. 23-33.

Donnay, G., Morimoto, N., Takeda, H. \& Donnay, J. D. H. (1964). Acta Cryst. 17, 1369-1373.

Dornberger-Schiff, K. (1959). Acta Cryst. 12, 173.

Dornberger-Schiff, K. (1964). Grundzüge einer Theorie von OD-Strukturen aus Schichten. Abh. Dtsch. Akad. Wiss. Berlin, Kl. Chem. No 3.

Dornberger-Schiff, K., Backhaus, K.-O. \& Ďurovič, S. (1982). Clays Clay Miner. 30, 364-374. 
Dornberger-Schiff, K. \& Farkas-Jahnke, M. (1970). Acta Cryst. A26, 24-34.

Ďurovič, S. (1979). Krist. Tech. 14, 1047-1053.

Ďurovič, S. (1992). International Tables for Crystallography, Vol. C, edited by A. C. J. Wilson, Section 9.2.2. Dordrecht: Kluwer Academic Publishers.

Ďurovič, S. (1994a). Ceramics-Silikáty, 38, 81-84.

Durovič, S. (1994b). Aperiodic '94. Proceedings of the International Conference on Aperiodic Crystals, edited by G. Chapuis \& W. Paciorek, pp. 595-599. Singapore: World Scientific.

Ďurovič, S. (1997). Modular Aspects of Minerals/EMU Notes in Mineralogy, Vol. 1, edited by S. Merlino, pp. 1-28. Budapest: Eötvös University Press.

Ďurovič, S. \& Weiss, Z. (1986). Bull. Minéral. 109, 15-29.

Ďurovič, S., Weiss, Z. \& Backhaus, K.-O. (1984). Clays Clay Miner. 32, 454-474.

D'yakonov, Yu. S. (1962). Sov. Phys. Crystallogr. 6, 499-500.

D'yakonov, Yu. S. (1963). Sov. Phys. Crystallogr. 6, 715-717.

Farkas-Jahnke, M. (1966). Acta Cryst. 21 A173.

Farkas-Jahnke, M. \& Dornberger-Schiff, K. (1970). Acta Cryst. A26, 35-41.

Fichtner-Schmittler, H. (1979). Krist. Tech. 14, 1079-1088.

Filut, M. A., Rule, A. C. \& Bailey, S. W. (1985). Am. Mineral. 70, 1298-1308.

Giuseppetti, G. \& Tadini, C. (1972). Tschermaks Mineral. Petrogr. Mitt. 18, 169-184.

Guggenheim, S. (1981). Am. Mineral. 66, 1221-1232.

Guggenheim, S. \& Bailey, S. W. (1975). Am. Mineral. 60, 1023-1029.

Guggenheim, S. \& Bailey, S. W. (1977). Am. Mineral. 62, 1158-1167.

Guggenheim, S. \& Bailey, S. W. (1978). Am. Mineral. 63, 186-187.

Guinier, A., Bokij, G. B., Boll-Dornberger, K., Cowley, J. M., Ďurovič, S., Jagodzinski, H., Khrisna, P., de Wolff, P. M., Zvyagin, B. B., Cox, D. E., Goodman, P., Hahn, Th., Kuchitsu, K. \& Abrahams, S. C. (1984). Acta Cryst. A40, 399-404.

Hahn, Th. \& Vos, A. (1996). International Tables for Crystallography, Vol. A, edited by Th. Hahn, Section 2.13. Dordrecht: Kluwer Academic Publishers.

Kogure, T. (1997). Mineral. J. 19, 155-164.

Kogure, T. \& Nespolo, M. (1999a). Acta Cryst. B. In the press.

Kogure, T. \& Nespolo, M. (1999b). Clays Clay Miner. Submitted.

Lipson, H. \& Taylor, C. A. (1958) Fourier Transforms and $X$-ray Diffraction. London: Bell.

Matsumoto, T. \& Wondratschek, H. (1979). Z. Kristallogr. 150, 181-198.

Matsumoto, T. \& Wondratschek, H. (1987). Z. Kristallogr. 189, 7-30.

Merlino, S. (1990). Period. Mineral. 59, 69-92.

Merlino, S. (1997). Modular Aspects of Minerals/EMU Notes in Mineralogy, Vol. 1, edited by S. Merlino, pp. 29-54. Budapest: Eötvös University Press.

Nespolo, M. (1998). Doctoral dissertation, University of Tokyo, Japan.

Nespolo, M. (1999). Mineral. J. 21. In the press

Nespolo, M. \& Kogure, T. (1998). Z. Kristallogr. 213, 4-12.

Nespolo, M., Takeda, H. \& Ferraris, G. (1997). Modular Aspects of Minerals/EMU Notes in Mineralogy, Vol. 1, edited by S. Merlino, pp. 81-118. Budapest: Eötvös University Press.

Nespolo, M., Takeda, H. \& Ferraris, G. (1998). Acta Cryst. A54, 348-356.

Nespolo, M., Takeda, H., Ferraris, G. \& Kogure, T. (1997). Mineral. J. 19, 173-186.

Ohta, T., Takeda, H. \& Takéuchi, Y. (1982). Am. Mineral. 67, 298-310.

Pabst, A. (1959). Z. Kristallogr. 112, 53-59.

Pabst, A. (1961). Z. Kristallogr. 115, 307-309.

Pauling, L. (1930). Proc. Natl Acad. Sci. USA, 16, 123-129.

Pavese, A., Ferraris, G., Ivaldi, G., Pischedda, V. \& Nespolo, M. (1998). 17th IMA General Meeting, Toronto, Canada. Abstract A102.

Pavese, A., Ferraris, G., Prencipe, M. \& Ibberson, R. (1997). Eur. J. Miner. 9, 1183-1190.

Radoslovich, E. W. (1961). Nature (London), 191, 67-68.

Ramsdell, L. S. (1947). Am. Mineral. 32, 64-82.

Rieder, M. (1968). Science, 160, 1338-1340.

Rieder, M., Cavazzini, G., D'Yakonov, Yu. S., FrankKamenetskii, V. A., Gottardi, G., Guggenheim, S., Koval', P. V., Müller. G., Neiva, A. M. R., Radoslovich, E. W., Robert, J. L., Sassi, F. P., Takeda, H., Weiss, Z. \& Wones, D. R. (1998). Can. Mineral. 36, 905-912.

Ross, M., Takeda, H. \& Wones, D. R. (1966). Science, 151, 191-193.

Rule, A. C., Bailey, S. W., Livi, K. J. T. \& Veblen, D. R. (1987). Am. Mineral. 72, 1163-1169.

Rumanova, I. M. \& Belov, N. V. (1961). Sov. Phys. Crystallogr. 5, 215-217.

Sadanaga, R. \& Takeda, H. (1964). Mineral. J. 4, 159-171.

Sadanaga, R. \& Takeda, H. (1969). J. Miner. Soc. Jpn, 9, 177-184. (In Japanese.)

Smith, J. V. \& Yoder, H. S. (1956). Mineral. Mag. 31, 209-235.

Takeda, H. (1961). Doctoral dissertation, University of Tokyo, Japan.

Takeda, H. (1967). Acta Cryst. 22, 845-853.

Takeda, H. (1971). Am. Mineral. 56, 1042-1056.

Takeda, H. \& Burnham, C. W. (1969). Mineral. J. 6, 102-109.

Takeda, H. \& Donnay, J. D. H. (1965). Am. Crystallogr. Assoc. Winter Meeting, Program and Abstracts, pp. 23-24.

Takeda, H., Haga, N. \& Sadanaga, R. (1971). Mineral. J. 6, 203-215.

Takeda, H. \& Horiuchi, H. (1971). J. Miner. Soc. Jpn, 10, 283-295. (In Japanese.)

Takeda, H. \& Mori, N. (1970). J. Crystallogr. Soc. Jpn, 12, 231-248. (In Japanese.)

Takeda, H. \& Morosin, B. (1975). Acta Cryst. B31, 2444-2452.

Takeda, H. \& Ross, M. (1995). Am. Mineral. 80, 715-724.

Takeda, H. \& Sadanaga, R. (1969). Mineral. J. 5, 434-449.

Takéuchi, Y. (1971). J. Miner. Soc. Jpn, 10, Spec. Issue No. 2, 87-99. (In Japanese.)

Takéuchi, Y. \& Haga, N. (1971). Miner. Soc. Jpn Spec. Pap. 1 , 74-87. (Proc. IMA-IAGOD Meetings '70, IMA Vol.)

Takéuchi, Y. \& Sadanaga, R. (1959). Acta Cryst. 12, 945-946.

Templeton, D. H. (1956). Acta Cryst. 9, 199-200.

Tokonami, M. (1966). Mineral. J. 4, 401-423.

Tokonami, M. \& Hosoya, S. (1965). Acta Cryst. 18, 908-916. 
Weiss, Z. \& Wiewióra, A. (1986). Clays Clay Miner. 34, 53-68. Wondratschek, H. (1976). Z. Kristallogr. 143, 460-470.

Zhukhlistov, A. P., Zvyagin, B. B. \& Pavlishin, V. I. (1988). Z. Kristallogr. 185, 624.

Zhukhlistov, A. P., Zvyagin, B. B. \& Pavlishin, V. I. (1990). Sov. Phys. Crystallogr. 35, 232-236.

Zhukhlistov, A. P., Zvyagin, B. B., Soboleva, S. V. \& Fedotov, A. F. (1973). Clays Clay Miner. 21, 465-470.

Zvyagin, B. B. (1962). Sov. Phys. Crystallogr. 6, 571-580.

Zvyagin, B. B. (1967). Electron Diffraction Analysis of Clay Mineral Structures. New York: Plenum Press.

Zvyagin, B. B. (1974). 2nd Eur. Crystallogr. Meet. Collect. Abstracts, Keszthely, pp. 162-164.
Zvyagin, B. B. (1985). Sov. Phys. Crystallogr. 32, 394-399.

Zvyagin, B. B. (1988). Comput. Math. Appl. 16, 569-591.

Zvyagin, B. B. (1997). Modular Aspects of Minerals/EMU Notes in Mineralogy, Vol. 1, edited by S. Merlino, pp. 345-372. Budapest: Eötvös University Press.

Zvyagin, B. B., Rabotnov, V. T., Sidorenko, O. V. \& Kotel'nikov, D. D. (1985). Izv. Akad. Nauk SSSR Ser. Geol. 5, 121-124. (In Russian.)

Zvyagin, B. B., Vrublevskaya, Z. V., Zhukhlistov, A. P., Sidorenko, O. V., Soboleva, S. V. \& Fedotov, A. F. (1979). High-Voltage Electron Diffraction in the Study of Layered Minerals. Moscow: Nauka. (In Russian.) 